\title{
Improvement of one-nucleon removal and total reaction cross sections in the Liège intranuclear-cascade model using Hartree-Fock-Bogoliubov calculations
}

\author{
Jose Luis Rodríguez-Sánchez, ${ }^{1,}$ Jean-Christophe David, ${ }^{1}$ Davide Mancusi, ${ }^{2}$ Alain Boudard, ${ }^{1}$ \\ Joseph Cugnon, ${ }^{3}$ and Sylvie Leray ${ }^{1}$ \\ ${ }^{1}$ IRFU, CEA, Université Paris-Saclay, F-91191 Gif-sur-Yvette, France \\ ${ }^{2}$ SERMA, CEA, Université Paris-Saclay, F-91191 Gif-sur-Yvette, France \\ ${ }^{3} A G O$ department, University of Liège, allée du 6 août 19, bâtiment B5, B-4000 Liège, Belgium \\ (Received 20 July 2017; revised manuscript received 16 October 2017; published 20 November 2017)
}

\begin{abstract}
The prediction of one-nucleon-removal cross sections by the Liège intranuclear-cascade model has been improved using a refined description of the matter and energy densities in the nuclear surface. Hartree-FockBogoliubov calculations with the Skyrme interaction are used to obtain a more realistic description of the radial-density distributions of protons and neutrons, as well as the excitation-energy uncorrelation at the nuclear surface due to quantum effects and short-range correlations. The results are compared with experimental data covering a large range of nuclei, from carbon to uranium, and projectile kinetic energies. We find that the new approach is in good agreement with experimental data of one-nucleon-removal cross sections covering a broad range in nuclei and energies. The new ingredients also improve the description of total reaction cross sections induced by protons at low energies, the production cross sections of heaviest residues close to the projectile, and the triple-differential cross sections for one-proton removal. However, other observables such as quadruple-differential cross sections of coincident protons do not present any sizable sensitivity to the new approach. Finally, the model is also tested for light-ion-induced reactions. It is shown that the new parameters can give a reasonable description of the nucleus-nucleus total reaction cross sections at high energies.
\end{abstract}

DOI: 10.1103/PhysRevC.96.054602

\section{INTRODUCTION}

Spallation reaction is a process in which a light projectile (proton, neutron, or light nucleus) with the kinetic energy from a few MeVs to several GeVs interacts with a heavy nucleus (e.g., lead) and causes the emission of a large number of hadrons (mostly neutrons) or some light fragments. This kind of reaction is usually described by using hybrid models consisting of an intranuclear-cascade (INC) stage followed by a statistical or dynamical de-excitation stage [1-3]. Here INC models, or more sophisticated ones based on the Boltzmann-Uehling-Uhlenbeck (BUU) and on VlasovUehling-Uhlenbeck (VUU) transport equations [4-7] as well as models from the family of quantum molecular dynamics (QMD) [8-10], are used to describe the collisions between target and projectile and the time evolution of the nuclear system before its thermalization. These models solve the transport equations numerically and their results are in good agreement with experimental data of spallation reactions at high and intermediate energies [2,9-12]. In this sense, INC models can be considered as a Monte Carlo method to solve the transport equations [13].

The nature of INC models is essentially classical, being typically assumed that nucleons are perfectly localized in phase space and that they are bound by a constant potential. In this approach, the nuclear collision is treated as successive relativistic binary hadron-hadron collisions separated in time and the positions and momenta of nucleons are followed as time evolves. Cross sections are determined from a set of

*jose.rodriguez-sanchez@cea.fr collision events taken at different impact parameters and for which nucleon positions and momenta are initially sampled for each participant nucleus.

These models are found to be the best tool to get more realistic descriptions of one-nucleon-removal cross sections for different reasons. On the one hand, we can follow in time all the particles participating in the nuclear collision, determining their multiple-scattering probabilities and final states. In other models, such as the distorted-wave Born approximation (DWBA) or distorted-wave impulse approximation (DWIA) [14,15], the evaluation of the multiple scattering at higher energies $(>300 \mathrm{MeV})$ is complicated by the possible production of pions and their propagation, which is not considered [16,17]. On the other hand, the dynamical models permit us to characterize the remnant in atomic and mass numbers, excitation energy, and angular momentum $[18,19]$. Consequently, this characterization allows one to calculate the de-excitation of the remnant that is dominated by the emission of light particles, such as neutrons and protons, and $\gamma$ emission, obtaining a realistic description of the reaction residues $[20,21]$.

During recent decades, it has been demonstrated that INC models coupled to de-excitation ones are able to describe many experimental observables [22]. Recently, some works have pointed that this kind of model describes reasonably inclusive measurements of one-nucleon-removal reactions on light nuclei, such as carbon or oxygen, at projectile energies below $120 \mathrm{MeV}$ [23-25] where, basically, one-nucleon removal reactions must fulfill two conditions simultaneously: Only one nucleon must be knocked out in the intranuclear-cascade process and the residual excitation energy of the remnant must be below the neutron and proton separation energies. However, 
these models fail in the description of inclusive measurements of one-nucleon-removal cross sections of medium and heavy nuclei [26,27]. This disagreement is especially surprising because one-nucleon-removal processes are associated with peripheral quasifree nucleon-nucleon reactions, where INC models should give a good description.

Recently, this discrepancy has been investigated in Ref. [26] using a simple version of the shell model to introduce some structural effects in the initial conditions of the nuclear surface, in particular, the neutron skin and the energy-density uncorrelation from quantum effects. It was demonstrated for two nuclei, ${ }^{40} \mathrm{Ca}$ and ${ }^{208} \mathrm{~Pb}$, that this refined description of the nuclear surface improves the description of one-nucleonremoval reactions. The need of structural effects in INC models for a realistic description of experimental observables was already pointed out in other works, for instance, to study photon-nucleus reactions [11,28,29] and the Primakoff effect [30], providing reasonable results for photoproduction yields.

To go further in the investigation of single-nucleonknockout reactions, we have used Hartree-Fock-Bogoliubov calculations [31] instead of the basic shell model to extend the previous work to all existing nuclei between the proton and neutron drip lines. In Sec. II, we describe the models as well as the improvements, and in Sec. III, we compare the model calculations with experimental data of different observables.

\section{MODEL DESCRIPTION}

\section{A. Liège intranuclear-cascade code}

Intranuclear cascade models [10,11,28,32-40] are commonly used for the description of nucleon-induced reactions at high energy ( $\gtrsim 100 \mathrm{MeV}$ ). In this context, it is assumed that the first stage of the reaction can be described as an avalanche of independent binary collisions. The INC scheme can be derived from the usual nuclear transport equations under suitable approximations [13] and its numerical solution can be efficiently tackled on today's computers. The INC model is essentially classical, with the addition of a few suitable ingredients that mimic genuine quantum-mechanical features of the initial conditions and of the dynamics: For instance, target nucleons are endowed with Fermi motion, realistic space densities are used, the output of binary collisions is random, and elementary nucleon-nucleon collisions are subject to Pauli blocking.

In this work we use the latest $\mathrm{C}++$ version of the INCL code [41], which is equivalent to the reference FORTRAN INCL 4.6 version [42] extended to nucleus-nucleus collisions. Hereafter, we will refer to this version as the INCL model, for simplicity. In this model, the hadron-nucleus or nucleus-nucleus reactions are modeled as a sequence of binary collisions between the nucleons (hadrons) present in the system. Hadrons move along straight trajectories until they undergo a collision with another hadron or until they reach the surface, where they eventually escape. During the cascade process, the particles are divided into participants and spectators. Participants are defined as particles that have collided with at least one other participant, while spectators are the other particles. Collisions between spectators are forbidden in order to eliminate the spontaneous boiling of the Fermi sea, which leads to particles that could escape from the target, even if the particle is left alone. This condition is used because the spontaneous boiling is a direct violation of the Pauli-exclusion principle. Strict Pauli blocking is also applied to the first collision to account for surface effects and for effects of the depletion of the Fermi sea according to Ref. [43]. For the subsequent collisions, we apply the Pauli principle according to the usual procedure by means of statistical blocking factors [2]. In addition, a consistent dynamical Pauli blocking is also used to reject unphysical results; see, for instance, Ref. [2] for details. There are some sophisticated models based on the BUU transport equation that also apply the Pauli blocking to the initial ground-state configuration since two nucleons could populate the same phase space [5]. However, we have seen that in our standard target preparation the probability of this kind of event is negligible and for this reason we do not use it. Finally, the latest version of the INCL also includes isospinand energy-dependent nucleus potentials calculated according to optical models [42] and isospin-dependent pion potentials [44]. Cluster emission is also possible via a dynamical phasespace coalescence algorithm [42].

Target and projectile density profiles are prepared at the first step of the simulation according to the mass number $(A)$ of the nucleus:

$$
\rho(r)= \begin{cases}\rho_{0} \frac{1}{1+\exp \left(\frac{r-R_{0}}{a}\right)} & \text { for } A>19 \\ \rho_{0} \frac{\left(1+\alpha(r / a)^{2}\right)}{\exp \left((r / a)^{2}\right)} & \text { for } 6<A \leqslant 19 \\ \rho_{0} \frac{1}{\exp \left((r / a)^{2}\right)} & \text { for } A \leqslant 6 .\end{cases}
$$

In the literature [45], these parametrizations are known as the Woods-Saxon, modified-harmonic-oscilator (MHO), and Gaussian density distributions, from top to bottom in Eq. (1) respectively. For the Woods-Saxon density distribution, the radius $\left(R_{0}\right)$ and the diffuseness parameter $(a)$ are taken from electron scattering measurements and parametrized according to Ref. [2]. For the other parametrizations, the parameters are taken from Ref. [45].

Note that in INCL the target and projectile preparations are different. For light projectile nuclei, those treated by INCL $(A \leqslant 18)$, the nucleon momenta are described with a Gaussian distribution considering the same rms momentum $\left(3 / 5 p_{F}\right)$ for protons and neutrons, with $p_{F}=270 \mathrm{MeV} / c$. For target nuclei, the initial nucleon momenta are uniformly distributed in hard Fermi spheres of radii $(2 Z / A)^{1 / 3} p_{F}$ for protons and $(2 N / A)^{1 / 3} p_{F}$ for neutrons (see Refs. [41] and [26] for more details). Moreover, for target nuclei, the nucleons are sampled in phase space, taking into account the correlations between kinetic energy and radius of the potential well [2]. The relation is such that the space density distribution is given by Eq. (1). Inspired by the properties of classical particle motion in a potential well, nucleons close to the Fermi energy travel farther out than those with small energies. Therefore, the radius is a function of the kinetic energy of the nucleons inside the potential well, such as $R=R(T)$ being $T$ the kinetic energy. For projectile nuclei, this kind of correlation is not considered. 
In INCL, each event is fired at a given impact parameter $b$, ranging from 0 to a distance $b_{\max }$ larger than the interaction distance of the two nuclei. Only the nucleons of the target that are outside the overlap region of the projectile and target densities are considered spectators. The other nucleons of the target and all the nucleons of the projectile are considered participants. If two nucleons approach each other at a distance less than a minimum distance, they interact. The minimum distance is calculated from an energy-dependent parametrization of the nucleon-nucleon interaction cross section for $p n, p p$, and $n n$ collisions [46].

At the end of the intranuclear cascade, an excited remnant is left. This nucleus typically relaxes by emitting low-energy particles or, if possible, by fissioning. The time scale for the second stage is typically much longer than that for the first one, which justifies the fact that de-excitation is not described by INC but by a different class of models which rely on statistical assumptions about the properties of the excited remnant. It is required to couple INC to a de-excitation model if one wishes to describe the production of reaction residues.

For the de-excitation step, we use the ABLA07 model [47], which describes the de-excitation of a nucleus emitting $\gamma$ rays, neutrons, light-charged particles, and intermediate-mass fragments (IMFs) according to Weisskopf's formalism [48]. For a more realistic description of the de-excitation, the separation energies and the emission barriers for charged particles are also considered according to the atomic mass evaluation from 2003 [49] and the Bass potential [50], respectively. The $\gamma$-ray emission is also considered according to Ref. [51], but note that it is more likely at the end of the de-excitation process when the excitation energy is below the particle separation energies. In addition, de-excitation by fission is described as a diffusion process above the fission barrier according to Refs. [52,53].

The coupling of these model calculations has been benchmarked in several works about nucleon-induced reactions on different nuclei between carbon and uranium at incident energies from a few MeVs to $3 \mathrm{GeV}$, providing a satisfactory description of many observables such as isotopic distributions of evaporation and fission residues [20,21,41,54-59], doubledifferential cross sections $[2,21,41,42,60]$, total reaction and fission cross sections [2,41,42,61-63], and pion production $[2,44,64,65]$.

\section{B. Mocking up quantum effects in the nuclear surface}

As we said previously, in INCL, nucleons have classical motion in a static potential well. Without entering the details, which can be found in Ref. [2], the most obvious consequence is that a nucleon below the Fermi level has a classical turning point, which is strictly correlated to its energy. However, this picture is not compatible with quantum motion and interaction effects. The simplest way to look at this problem is to consider that a nucleon inside the Fermi sea is in fact described by a wave function. One immediate consequence is that the nucleon my be found beyond its classical turning point. Or to phrase it more illustratively, the turning point is no more a fixed point but may fluctuate around its classical value. This point of view was adopted by the authors of Ref. [26], who spoke about fuzziness (of the classical turning point). To evaluate the importance of this correction to be brought to the INCL model and to implement it, they proposed to consider a shell model of independent nucleons. The parameters of the shell model were determined such that the space density profile is the same as the one of the INCL model. Looking at the wave functions, one can see the distribution of the nucleons beyond their classical point. Instead of classical points, the distribution of the energy of the nucleons can be analyzed. Without entering into details, it can be seen that, for a given location in the surface, the energy of the nucleons is larger in the shell model than in the INCL model. In addition, a "fuzziness parameter" $f$ was introduced for somehow modulating the effect (for $f=0$, the pure INCL model is recovered).

\section{Hartree-Fock-Bogoliubov approach}

In previous studies using INC approaches, the authors were interested in the isotopic spallation production cross sections from stable nuclei [2,42], where the neutron and proton densities were taken as Woods-Saxon profiles with parameters set to reproduce the charge density measured by electron scattering. However, one-nucleon-removal reactions are expected to be more sensitive to the relative densities of neutrons and protons at the surface of the nucleus. Therefore, in the present work, we adopt the same approach as in Ref. [26], but we use the single-particle wave functions from Hartree-Fock-Bogoliubov calculations performed with the HFBRAD code [31] considering the Skyrme Sly5 interaction [66]. We decided to utilize this approach because it provides a reasonable description of the proton and neutron density radii $[27,67,68]$. The HFBRAD calculations were performed for all nuclei between the proton and neutron drip lines and an example of these calculations is shown in Fig. 1, where we represent the skin thickness as a function of the atomic and neutron number of the nucleus. As can be seen in the figure, neutron-rich nuclei have larger skin thickness as expected.

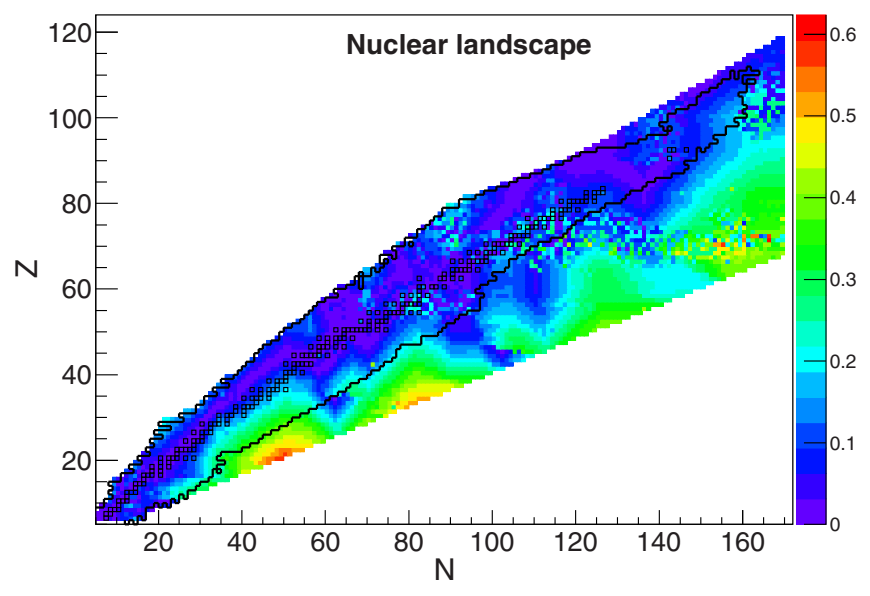

FIG. 1. Skin thickness in fm (defined as $\left|R_{n}-R_{p}\right|$ only to avoid negative values) calculated with the HFBRAD model [31] for nuclei between the proton and neutron drip lines defined according to Ref. [69]. The open squares represent the stable nuclei and the solid contour line indicates the region of known nuclei. 
We would like to use the HFBRAD proton and neutron densities as inputs for our INCL. However, the particle densities cannot be given by an arbitrary function, as explained in Ref. [26]. Following the previous approach (see Ref. [26]), we have fitted the density distributions with the Woods-Saxon equation for nuclei with mass number larger than $A=19$. For nuclei with mass numbers between $A=6$ and $A=19$, we have fitted the density distributions with the MHO equation [see Eq. (1)], respectively.

The HFBRAD model also provides us with a decomposition of the local density as a function of the radial position in terms of the various shells. At each position, we can construct the presence probabilities according to

$$
p_{n j}(r)=g_{n j} \rho_{n j}(r) / \rho(r),
$$

where the occupation numbers $g_{n j}$ are given for protons $(Z)$ and neutrons $(N)$ by

$$
g_{n j}= \begin{cases}2 j+1 & \text { if } E_{n j}<E_{\mathrm{F}} \\ Z(N)-\sum_{E_{n j}<E_{\mathrm{F}}} g_{n j} & \text { if } E_{n j}=E_{F}, \\ 0 & \text { if } E_{n j}>E_{F}\end{cases}
$$

assuming that the shells are filled from the bottom of the potential well up to the Fermi level $\left(E_{\mathrm{F}}\right) . \rho_{n j}(r)$ denotes the radial density profile for each shell described as

$$
\rho_{n j}(r)=4 \pi r^{2}\left|R_{n j}(r)\right|^{2},
$$

where $R_{n j}(r)$ are the radial eigenfunctions, and $\rho(r)$ represents the total density

$$
\rho(r)=\sum_{n j} g_{n j} \rho_{n j}(r) .
$$

With these simple ingredients, one can estimate the energy density at the surface of the nucleus, assuming that the knockout probability is proportional to the local density of the shell orbital and that the excitation energy of the hole $\left(E_{n j}^{*}\right)$ is given by its depth in the potential well according to

$$
E_{n j}^{*}=E_{\mathrm{F}}-E_{n j},
$$

where $E_{n j}$ are the eigenvalues of the holes with quantum numbers $n$ and $j$.

For the characterization of the energy density at the surface of the nucleus, we only need to study the probability that the excitation energy associated with the hole created after the one-nucleon knockout does not surpass the neutron binding energy $\left(S_{n}\right)$ of the remnant nucleus, which reads

$$
P_{E^{*}<S_{n}}(r)=\sum_{n j} p_{n j}(r) \Theta\left(S_{n}-E_{n j}^{*}\right),
$$

where $\Theta$ is the Heaviside function.

This method was used in Ref. [26] to constrain the fuzziness parameter $f$ that accounts for the energy fluctuations at the surface of the nucleus. As mentioned in Sec. II A, in INCL the nucleons move in a square-well potential whose radius $R(T)$ depends on the nucleon kinetic energy. The fuzziness parameter $f$ introduces random fluctuations in $R(T)$, in such a way that the space and momentum densities are still given by $\rho(r)$ and by a uniform Fermi sphere, respectively, and if $f=0$ the fluctuations are suppressed, recovering the standard
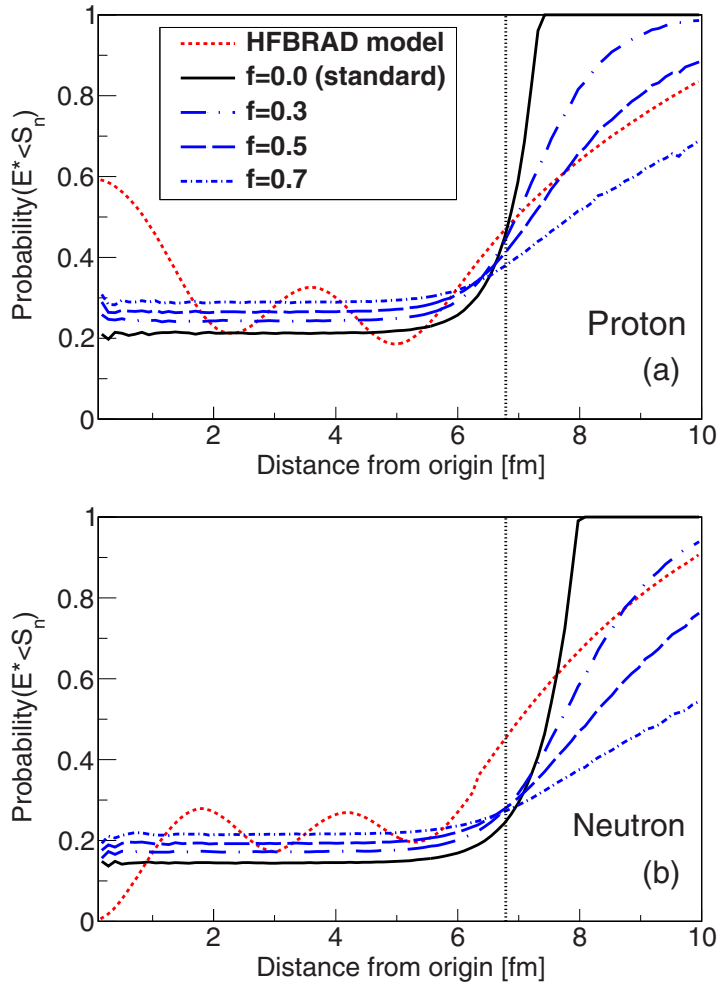

FIG. 2. Probability that a proton (a) or neutron (b) hole in ${ }^{208} \mathrm{~Pb}$ results in an excitation energy smaller than the neutron separation energy, as a function of the distance of the hole from the center of the nucleus. The dotted line corresponds to the HFBRAD calculations. The other lines represent the INCL initial conditions for different values of the fuzziness parameter $f$ defined in Ref. [26], where the standard condition corresponds to $f=0$. One-nucleon removal is dominated by impact parameters to the right of the vertical dotted line.

sharp correlation of INCL. Therefore, the construction of the fuzzy nucleus is analogous to the standard algorithm [2].

In Figs. 2 and 3, we compare the results obtained from the HFBRAD model (dotted line) with INCL calculations, considering different values of $f$ for proton [Figs. 2(a) and 3(a)] and neutron [Figs. 2(b) and 3(b)] knockout reactions on ${ }^{208} \mathrm{~Pb}$ and ${ }^{25} \mathrm{Mg}$, respectively. This comparison confirms that the excitation energy associated with the ejection of one nucleon is underestimated by the standard INCL (solid line), as pointed out previously in Ref. [26]. The HFBRAD calculations also allow us to constrain the fuzziness parameter $f$, which is taken to be $f=0.5$ for protons and $f=0.3$ for neutrons. The same calculations were performed for other nuclei (not shown), resulting in the same values obtained for ${ }^{208} \mathrm{~Pb}$ and ${ }^{25} \mathrm{Mg}$. Whereas those values could be slightly different from one target to another, in Sec. III, nevertheless, it will be shown that these constraints of the fuzziness parameter allow us to describe one-nucleon-removal cross sections with high precision.

In summary, the fuzziness parameter allows us to overcome the underestimation of the excitation energies at the surface of the nucleus, which is a general failure in INC approaches and other models based on the Glauber picture. The need 

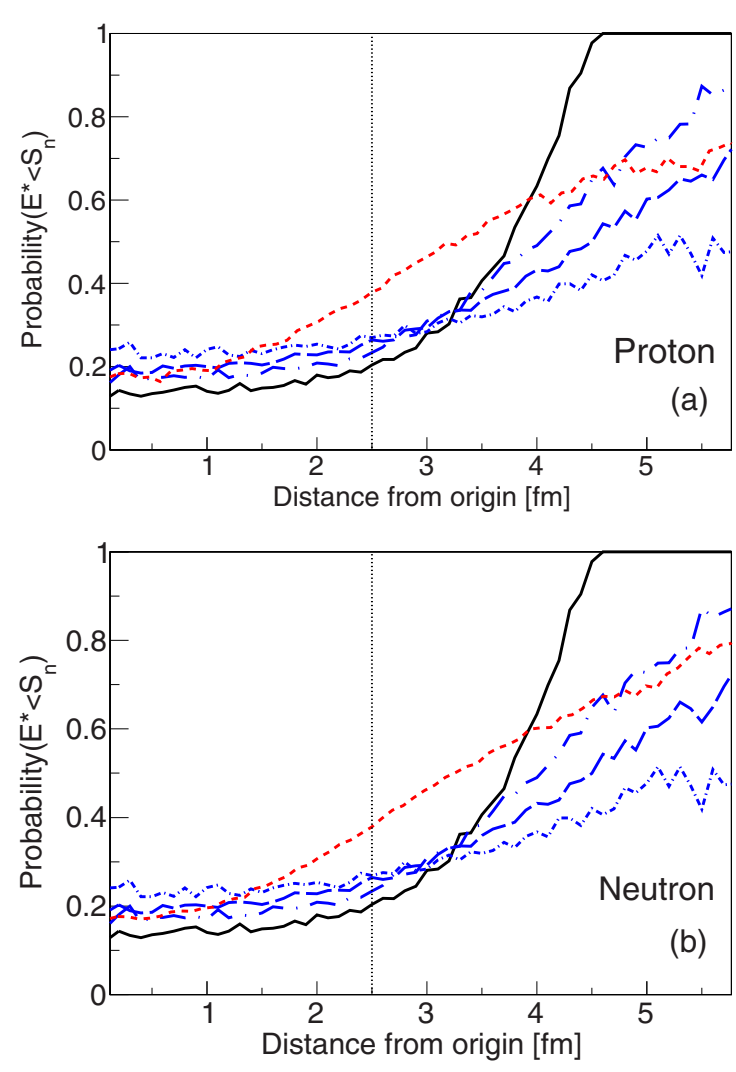

FIG. 3. Same as Fig. 2, but for ${ }^{25} \mathrm{Mg}$.

to increase the excitation energy for one- and multiplenucleon removal was already pointed in many works using fragmentation reactions on stable nuclei at high energies by comparing the experimental data with the abrasion-model predictions of Ref. [70]. In particular, the excitation-energy distributions for one- and multiple-proton-knockout reactions were artificially increased by a factor of two to reproduce the experimental cross sections [70-72]. Therefore, these phenomenological findings support our constraints.

\section{RESULTS}

As in Ref. [26], we have refined the description of the initial conditions of INCL using the parametrizations of the Hartree-Fock-Bogoliubov calculations described above. The neutron and proton density profiles calculated with the HFBRAD model have been introduced in INCL together with the energy density fluctuations at the nuclear surface. We have checked that as demonstrated in Ref. [26], some experimental observables, such as the double-differential cross sections for particle production or the isotopic distribution of fragments far from the projectile, are not sensitive to the new refined initial conditions. The new ingredients in INCL should only affect the production of nuclear fragments close to the projectile, in particular, one-nucleon-removal cross sections. Therefore, we compare our calculations with experimental data of one-neutron- and one-proton-removal cross sections and with isotopic cross sections of fragments produced close to the projectile. In addition, to go a step forward with respect to our previous work, we also compare our calculations with triple- and quadruple-differential cross sections of coincident knocked out protons measured in spallation reactions.

After the modifications introduced at the surface of the nucleus, we have to validate the new approach for total reaction cross sections induced by nucleons only to be sure that the new parameters do not affect this observable and also because it was not studied in our previous work [26]. The present work is also utilized to revise the nuclear density parametrization of light-ion projectiles introduced in INCL ++ recently for the description of nucleus-nucleus collisions because, as explained in Sec. II A, target and projectile nuclei preparations are different. For the benchmark, we will use the total reaction cross sections measured in carbon- and beryllium-induced reactions on different nuclei.

\section{A. One-nucleon-removal cross sections}

A complete analysis of the refined initial conditions in INCL was carried out in Ref. [26], mimicking the shell model. Here, we do the same using the HFBRAD model and only discuss the most important results. First, the introduction of the neutron skin increases (decreases) the neutron (proton) removal cross sections because it increases the neutron density with respect to the proton one at the surface of the nucleus [73], favoring the neutron knockout probability. Second, the fluctuations in the energy density at the surface of the nucleus increase the excitation energy of the remnant nuclei, reducing the one-neutron- and proton-removal cross sections.

In Figs. 4 and 5, we compare our new calculations (dashed lines) with experimental one-neutron- and one-proton-removal cross sections (solid circles) for the reactions $p+{ }^{40} \mathrm{Ca}[74,75]$ (Figs. 4(a) and 4(b), respectively) and $p+{ }^{208} \mathrm{~Pb}[76,77]$ (Figs. 5(a) and 5(b), respectively). These cross sections are displayed as a function of the proton kinetic energy. We also show the standard INCL calculations (solid lines) and the results obtained from the shell model (dotted lines) used previously [26]. As expected, the standard INCL calculations overestimate the experimental data for both nucleon removal channels. However, the calculations obtained with the shell and Hartree-Fock-Bogoliubov models provide a better description of the experimental data for $p+{ }^{40} \mathrm{Ca}$. We could also point out that the shell and Hartree-Fock-Bogoliubov models give very similar results for the cross sections because of two simple reasons. First, in both calculations we are using the same values for the fuzziness parameters $(f=0.5$ for protons and $f=0.3$ for neutrons). Second, both models provide similar results for the skin thickness as listed in Table I. The shell model predicts for ${ }^{40} \mathrm{Ca}$ a neutron skin thickness of $-0.08 \mathrm{fm}$ and the HFBRAD model gives a value of $-0.07 \mathrm{fm}$.

However, for ${ }^{208} \mathrm{~Pb}$ we can see that the shell model overestimates the cross sections while the new calculations provide a better description. Here, the difference between these calculations is because the shell and Hartree-FockBogoliubov models predict different neutron skin thicknesses: 0.27 and $0.12 \mathrm{fm}$, respectively. This fact leads to a good description of the neutron-removal cross sections, as shown in Fig. 5(a). In Fig. 5(b), one can also notice that the new 

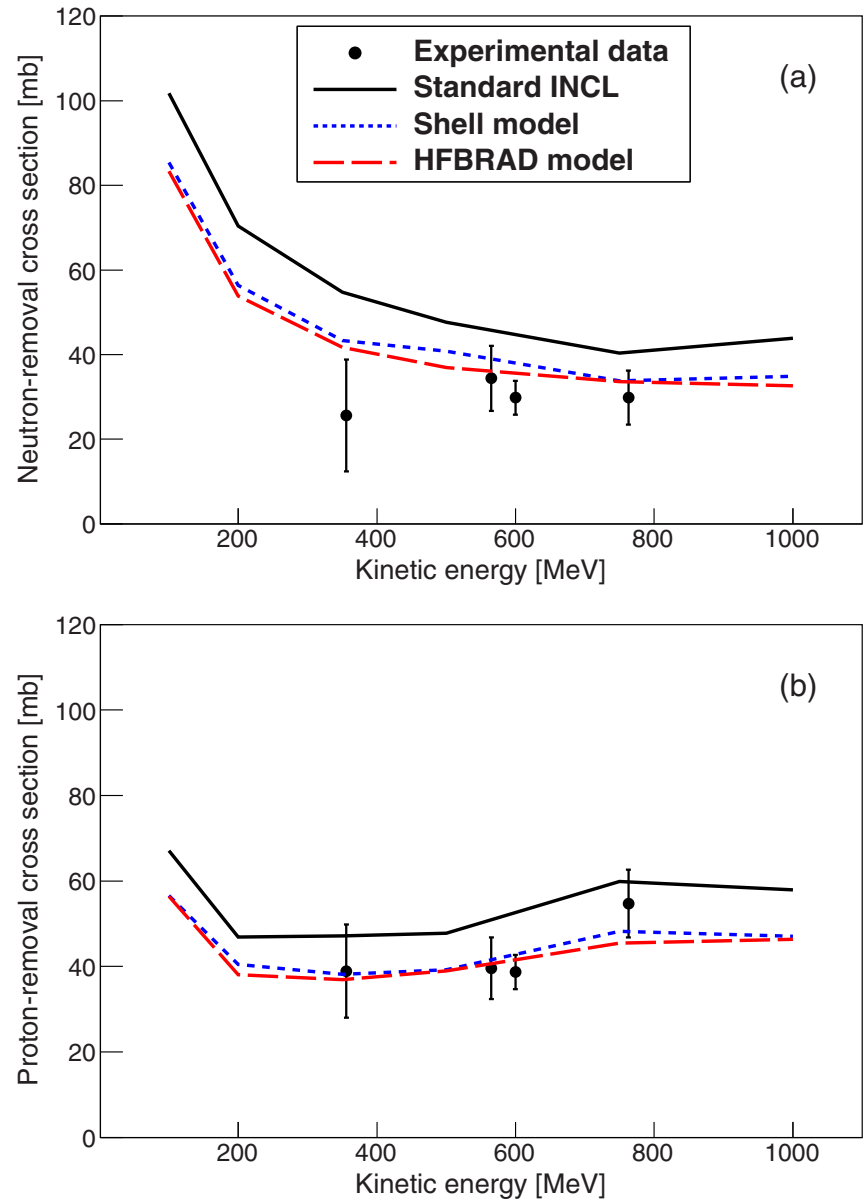

FIG. 4. One-neutron (a) and one-proton (b) removal cross sections as a function of the kinetic energy for the reaction $p+{ }^{40} \mathrm{Ca}$. Data taken from Refs. [74,75]. The solid line corresponds to standard INCL calculations and the short- and long-dashed lines represent INCL calculations using the shell model and Hartree-Fock-Bogoliubov calculations for the proton and neutron density profiles together with the surface fuzziness, respectively.

calculations are a bit closer to the experimental data because the diffuseness parameter predicted by the HFBRAD model for the proton radial density is smaller than the value used with the shell-model calculations $(\sim 0.54 \mathrm{fm})$, which was taken from the experimental charge density parametrization used in the standard INCL [26].

In Fig. 6, we compare our new calculations (dashed lines) and the standard INCL ones (solid lines) with experimental one-neutron- and one-proton-removal cross sections of different stable nuclei from ${ }^{12} \mathrm{C}$ to ${ }^{74} \mathrm{Ge}$ (solid circles). The experimental data and the calculations are displayed as a function of the proton kinetic energy. For a better understanding of these results, in the figures we also display calculations considering the proton and neutron density profiles from the Hartree-FockBogoliubov model and the fuzziness parameters set to zero (dotted lines). In the figure, we can see that the neutron skin reduces the one-proton-removal cross sections with respect to standard INCL calculations, while one-neutron-removal cross sections do not suffer big changes. When the two refinements are simultaneously applied, the effect of surface fuzziness
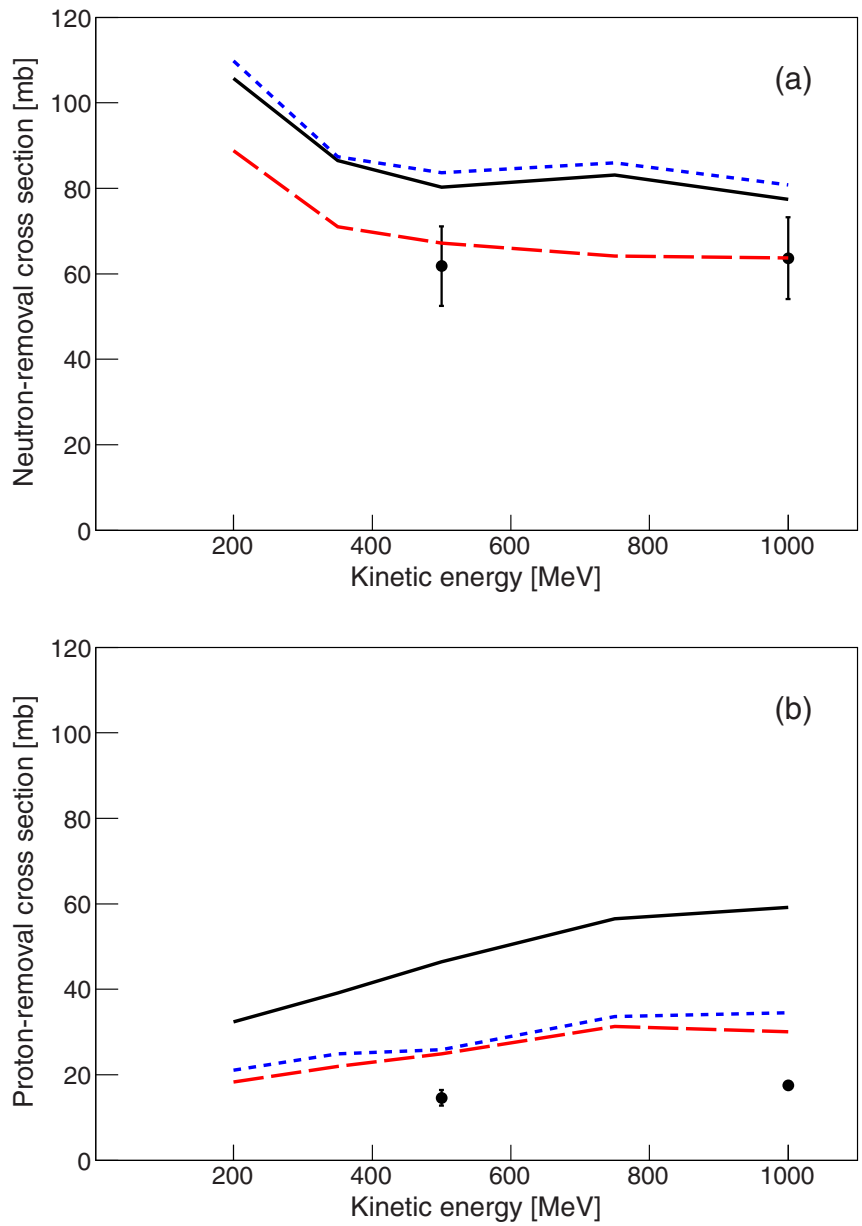

FIG. 5. Same as Fig. 4, but for the reaction $p+{ }^{208} \mathrm{~Pb}$. Experimental data taken from Refs. [76,77].

decreases the value of the cross sections for both channels, resulting in a better agreement with the experimental data, in particular, for the one-proton-removal cross sections.

Finally, in Fig. 7, we also overlap our new calculations (open triangles and open circles) and the standard INCL ones (open squares) with experimental one-neutron- and

TABLE I. Optimal parameters for Woods-Saxon densities fitting the results of shell-model [26] and HFBRAD [31] calculations. The skin and halo values are differences of the neutron and proton parameters, $\left(R_{n}-R_{p}\right)$ and $\left(a_{n}-a_{p}\right)$, respectively. All values are in $\mathrm{fm}$.

\begin{tabular}{lccccc}
\hline \hline Nucleus & Model & & Neutron & Proton & Skin/halo \\
\hline${ }^{40} \mathrm{Ca}$ & Shell model & $\mathrm{R}_{0}$ & 3.57 & 3.64 & -0.08 \\
& & $\mathrm{a}$ & 0.49 & 0.51 & -0.02 \\
& HFBRAD & $\mathrm{R}_{0}$ & 3.50 & 3.57 & -0.07 \\
& & $\mathrm{a}$ & 0.48 & 0.52 & -0.04 \\
${ }^{208} \mathrm{~Pb}$ & \multirow{2}{*}{ Shell model } & $\mathrm{R}_{0}$ & 6.98 & 6.71 & 0.27 \\
& & $\mathrm{a}$ & 0.55 & 0.46 & 0.09 \\
& \multirow{2}{*}{ HFBRAD } & $\mathrm{R}_{0}$ & 6.76 & 6.64 & 0.12 \\
& & $\mathrm{a}$ & 0.54 & 0.45 & 0.09 \\
\hline \hline
\end{tabular}



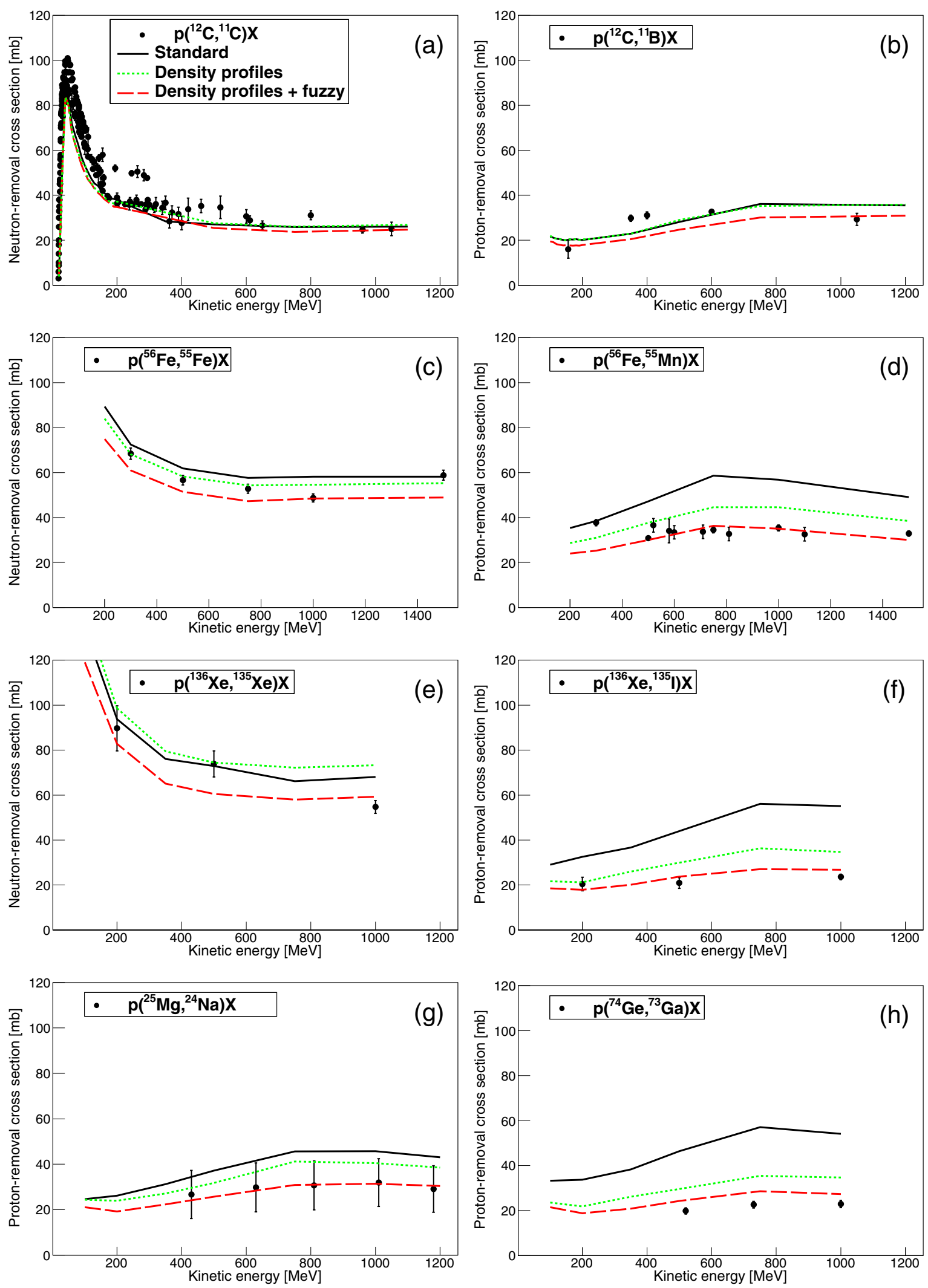

FIG. 6. One-neutron- and one-proton-removal cross sections as a function of the kinetic energy for different nuclei such as ${ }^{12} \mathrm{C}$ [panels (a) and (b)], ${ }^{56} \mathrm{Fe}$ [panels (c) and (d)], and ${ }^{136} \mathrm{Xe}$ [panels (e) and (f)], respectively. Panels (g) and (h) show the proton-removal cross sections for ${ }^{25} \mathrm{Mg}$ and ${ }^{74} \mathrm{Ge}$ as a function of the kinetic energy, respectively. Data taken from Refs. [55,78-84]. The solid line corresponds to standard INCL calculations. The dotted and dashed lines represent calculations assuming only the Hartree-Fock-Bogoliubov proton and neutron density profiles and considering simultaneously the density profiles and the energy fluctuations, respectively. 

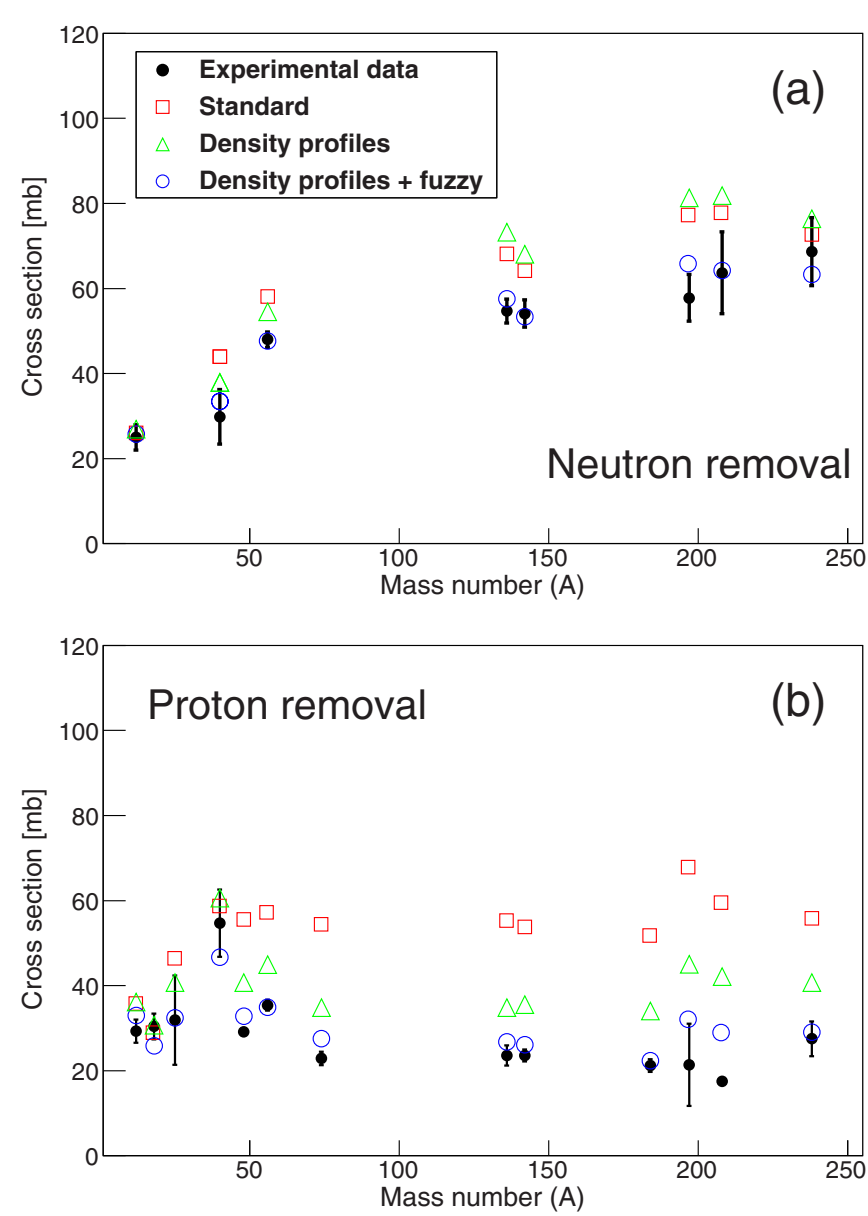

FIG. 7. One-neutron (a) and one-proton (b) removal cross sections from proton-induced reactions at kinetic energies of $1 \mathrm{GeV}$ as a function of the target mass number for nuclei from ${ }^{12} \mathrm{C}$ to ${ }^{238} \mathrm{U}$ (solid circles). Data taken from Refs. [55,75,77,79,81,84-87]. The open squares represent the standard INCL calculations and the open triangles correspond to calculations taking into account the proton and neutron density profiles obtained with the HFBRAD model. The open circles correspond to calculations where the two refinements, density profiles and surface fuzziness, are simultaneously applied.

one-proton-removal cross sections (solid circles) obtained in proton-induced reactions on stable nuclei from ${ }^{12} \mathrm{C}$ to ${ }^{238} \mathrm{U}$ at energies around $1 \mathrm{GeV}$. Considering only the proton and neutron density profiles from the HFBRAD model, the predictions are globally similar to the standard ones for one neutron removal. For the one-proton-removal channel, the cross section is roughly rescaled by a factor of $\sim 0.6$. When the two refinements are applied, the effect of surface fuzziness reduces both cross sections and brings them in better agreement with the trend shown by the experimental data.

The remarkable agreement between our predictions and the experimental data in Fig. 7, including the details of the dependence with the mass number, strongly emphasizes the well-foundedness of the Hartree-Fock-Bogoliubov model for describing the details of the nuclear surface properties. This agreement is also derived from the capacity of the INCL model to describe nucleon-nucleon collisions and, more precisely in this case, how the latter are avoided.

\section{B. Quadruple- and triple-differential cross sections for coincident proton emission}

The new description of the nuclear surface could also affect the emission of particles from peripheral collisions. In our previous work [26], we investigated this effect using doubledifferential cross sections for the production of neutrons and protons in spallation reactions at energies around $1 \mathrm{GeV}$. We observed that the new ingredients only broaden the peak from quasielastic charge-exchange reactions; this fact was attributed to the energy fluctuations increased at the
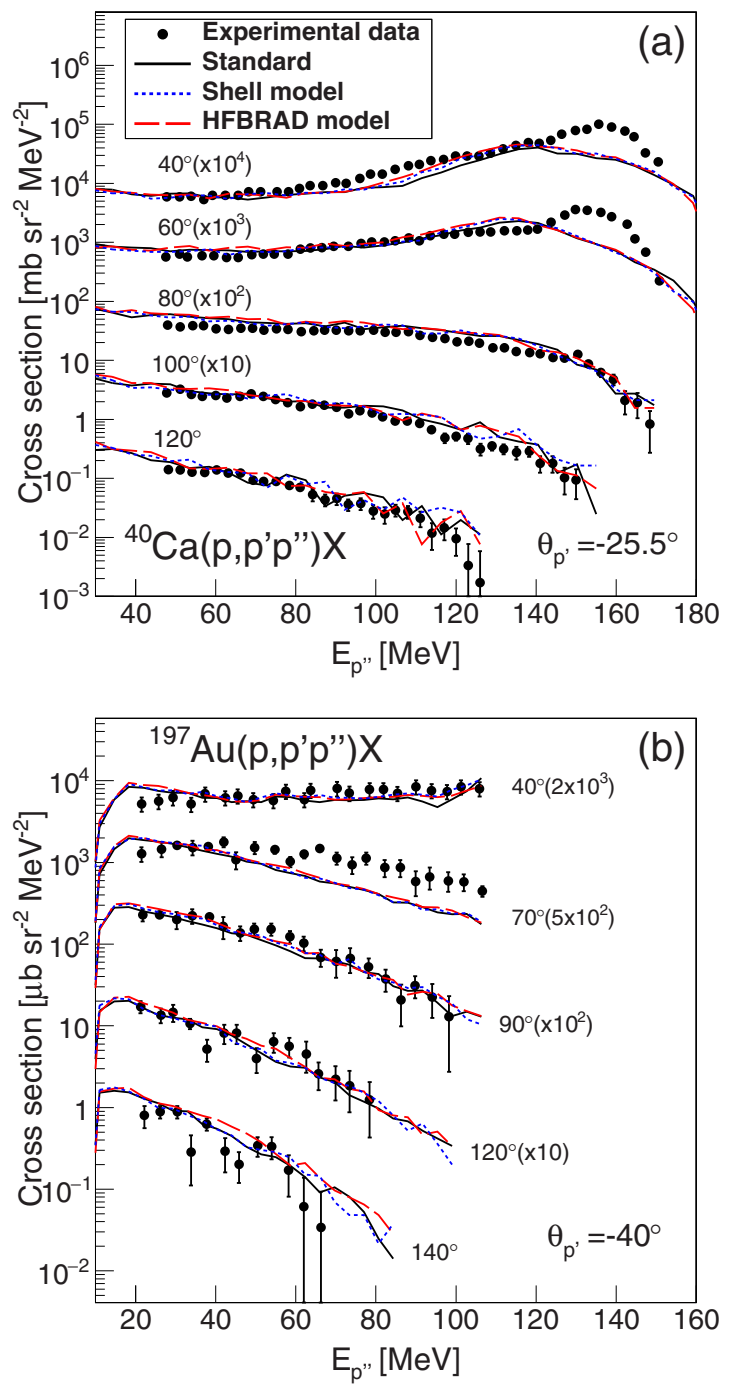

FIG. 8. (a) Quadruple-differential cross sections for two coincident protons emitted in the reaction $p+{ }^{40} \mathrm{Ca}$ at $392 \mathrm{MeV}$ [17]. The values for the detection angles of the second proton $\left(p^{\prime \prime}\right)$ are given close to the corresponding curves. Note the displacement factors for the purpose of display. The experimental data is compared with the standard INCL (solid lines), shell-model (dotted lines), and HFBRAD (dashed lines) calculations. (b) Same as (a) but for the reaction $p+{ }^{197} \mathrm{Au}$ at $200 \mathrm{MeV}$. Experimental data taken from Ref. [88]. 


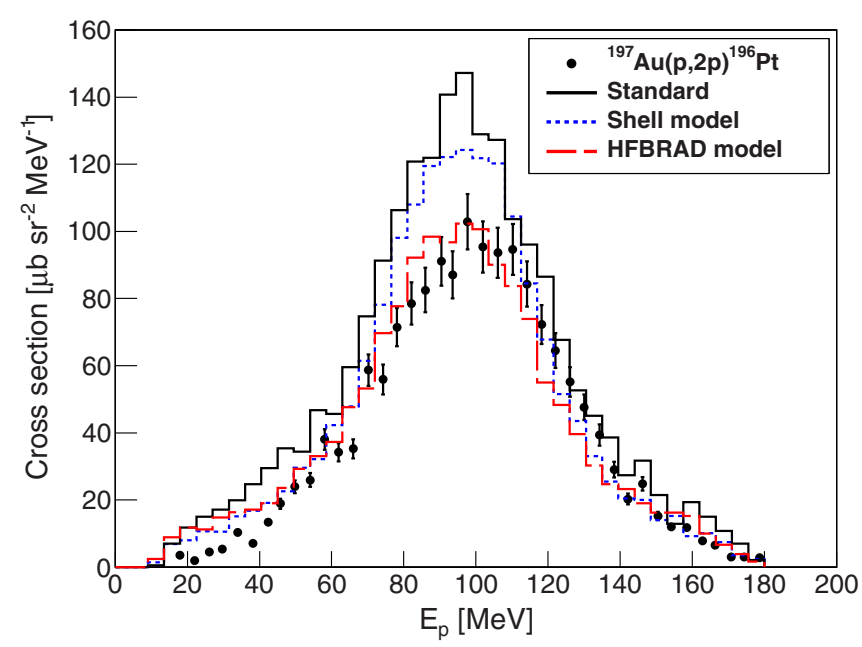

FIG. 9. Triple-differential cross sections for two coincident protons emitted in the reaction ${ }^{197} \mathrm{Au}(p, 2 p){ }^{196} \mathrm{Pt}$ at $200 \mathrm{MeV}$ [88]. Detection angles of the primary and secondary protons were taken around average values of $-40^{\circ}$ and $40^{\circ}$, respectively. The experimental data are compared with the standard INCL (solid lines), shell-model (dotted lines), and HFBRAD (dashed lines) calculations.

target surface. However, this observable does not permit us to have complete control over the number of knocked out nucleons in the collision. For this reason, in this work we use quadruple-differential cross sections of two coincident protons produced in proton-induced reactions on ${ }^{40} \mathrm{Ca}$ at 392 [17], and quadruple- and triple-differential cross sections of two coincident protons produced in proton-induced reactions on ${ }^{197} \mathrm{Au}$ at $200 \mathrm{MeV}$ [88]. We have selected this set of experimental data taking into account the study of onenucleon-removal cross sections performed in Sec. III A, where one could conclude that the effects from the new approach are more prominent for medium and heavy nuclei at projectile energies above $200 \mathrm{MeV}$.

In Fig. 8(a), we compare our calculations with experimental quadruple-differential cross sections obtained for the reaction ${ }^{40} \mathrm{Ca}\left(p, p^{\prime} p^{\prime \prime}\right) \mathrm{X}$. The comparison was carried out while taking into account the experimental selection. The primary proton $\left(p^{\prime}\right)$ was selected at a fixed forward angle of $-25.5^{\circ}$ and at a kinetic energy of $(220 \pm 20) \mathrm{MeV}$, while the second proton $\left(p^{\prime \prime}\right)$ was chosen according to the angles displayed in the figure. For the reaction ${ }^{197} \mathrm{Au}\left(p, p^{\prime} p^{\prime \prime}\right) \mathrm{X}$ [see Fig. 8(b)], the primary proton was set up at a fixed forward angle of $-40^{\circ}$ and at a kinetic energy of $(70 \pm 8) \mathrm{MeV}$. In both figures, we can see that standard INCL calculations (solid lines) provide a rather good description of this set of experimental data. One can also observe that the new approach, where the nuclear surface is shaped according to shell-model (dotted lines) and HFBRAD (dashed lines) calculations, does not provide a sizable improvement with respect to the standard INCL. This fact could indicate that this kind of observable, as well the double-differential cross sections, is mostly dominated by the nucleon-nucleon kinematics and by the rescattering of the particles involved in the collision [89]. Therefore, we can conclude that this observable under the experimental conditions explained above does not provide a good constraint
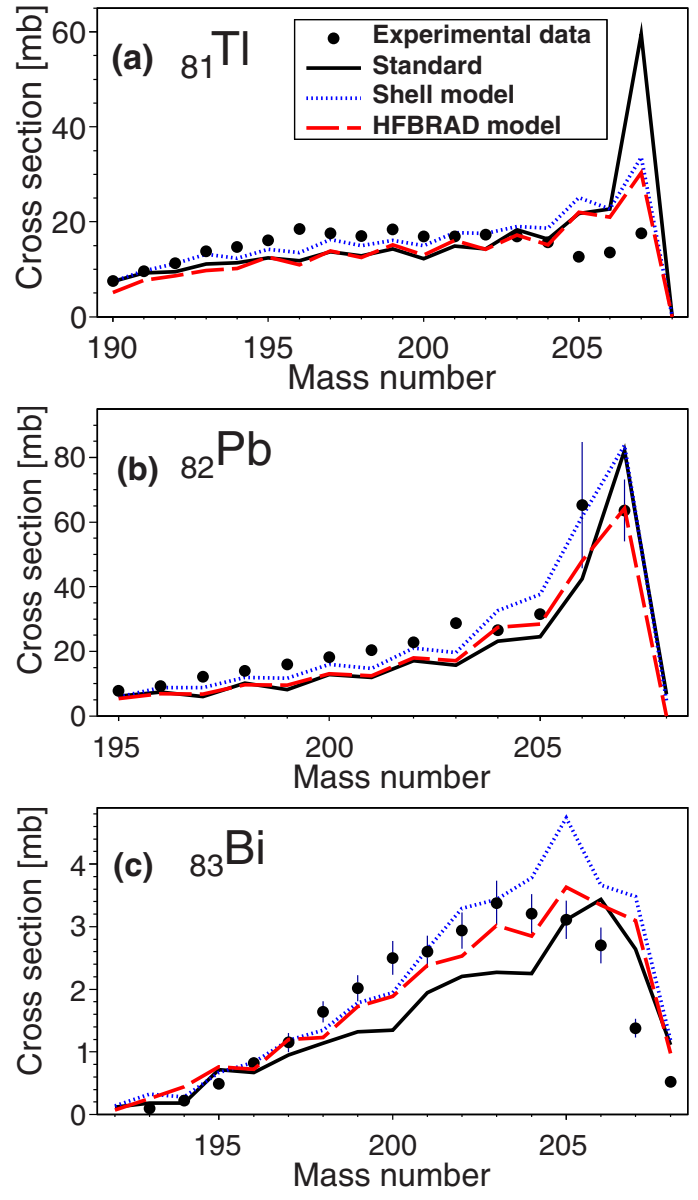

FIG. 10. Isotopic distributions of the $Z=81$ (a), $Z=82$ (b), and $Z=83$ (c) residues produced in $p+{ }^{208} \mathrm{~Pb}$ at $1 \mathrm{GeV}[77,90]$. The experimental data are compared with the standard INCL (solid lines), shell-model (dotted lines), and HFBRAD (dashed lines) calculations.

to study structural effects at the surface of the nucleus. We can also see that the calculations do not reproduce the peaks found at small angles in the reaction ${ }^{40} \mathrm{Ca}\left(p, p^{\prime} p^{\prime \prime}\right) X$ [see Fig. 8(a)]. The underestimation of these peaks could be related with proton emission after charge-exchange reactions. This fact was also observed in double-differential cross sections for particle production at small angles $[2,26]$.

However, the study of triple-differential cross sections for the reaction ${ }^{197} \mathrm{Au}(p, 2 p){ }^{196} \mathrm{Pt}$ at $200 \mathrm{MeV}$ [88], where the final residue is identified in order to select single-proton removal reactions, leads to different conclusions. As shown in Fig. 9, this observable is sensitive to the approach used to shape the nuclear surface. On the one hand, we can see that standard INCL calculations (solid line) overestimate the cross sections as also observed in Sec. III A for one-proton removal. On the other hand, calculations based on the shell (dotted line) and HFBRAD (dashed line) models improve the description of the experimental cross sections. In this case, one can observe that the nuclear surface description according the HFBRAD model provides a better agreement with the experimental data, especially at kinetic energies around $100 \mathrm{MeV}$. This fact is attributed to the value of the diffuseness parameter for the 

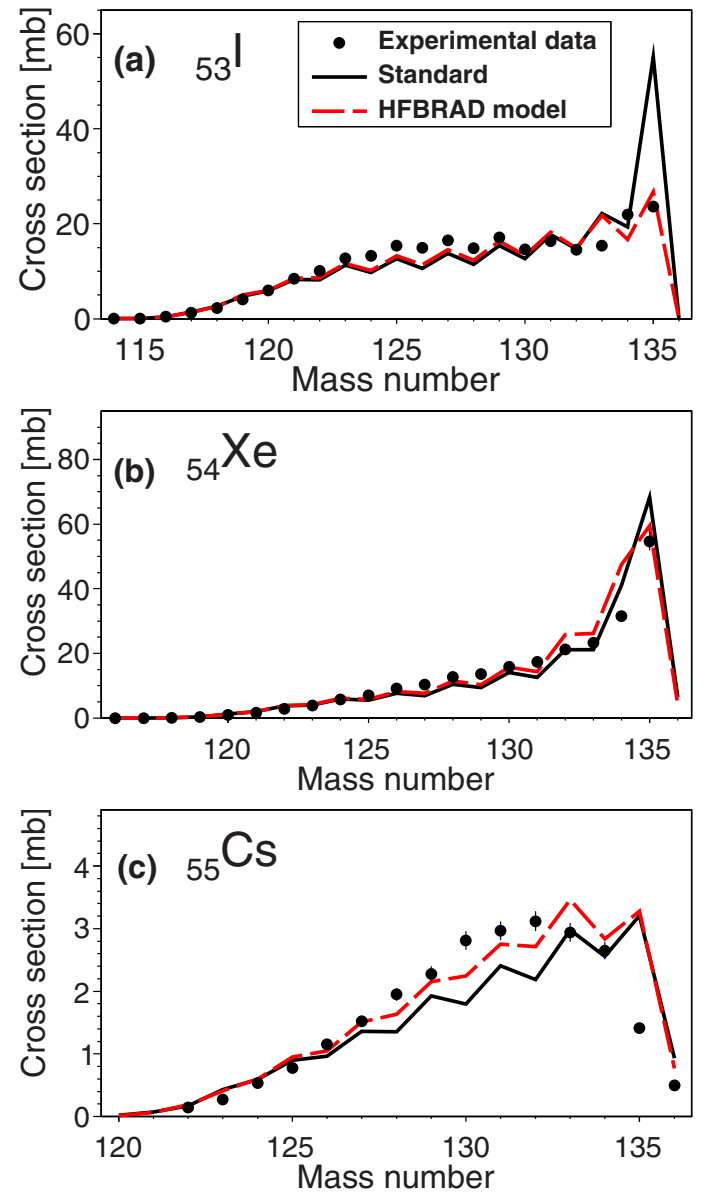

FIG. 11. Isotopic distributions of the $Z=53$ (a), $Z=54$ (b), and $Z=55$ (c) residues produced in $p+{ }^{136} \mathrm{Xe}$ at $1 \mathrm{GeV}$ [81]. The experimental data are compared with the standard INCL (solid lines) and HFBRAD (dashed lines) calculations.

proton density $\left(a_{p}\right)$. For the shell model, we are taking values from the experimental parametrization implemented in INCL whose result for ${ }^{197} \mathrm{Au}$ is $a_{p}=0.54 \mathrm{fm}$, while HFBRAD calculations predict a value of $0.42 \mathrm{fm}$. This fact reduces the probability of single-proton-knockout reactions at the nuclear surface and explains the results shown in the figure. Therefore, this observable allows us to demonstrate that the HFBRAD model is better to shape the nuclear surface and also validates our approach.

\section{Isotopic distributions}

One of the most important benchmarks for model calculations is the comparison with the isotopic distributions of nuclear residues. In the present work, we focus our attention on the cross sections of nuclei produced close, in atomic and mass number, to the projectile because their production is more sensitive to the new refined initial conditions of INCL. The effect of the skin thickness and surface fuzziness is illustrated in Fig. 10, where we display the isotopic cross sections for the heaviest residues produced in the reaction $p+{ }^{208} \mathrm{~Pb}$ at $1 \mathrm{GeV}$.

For the production of $\mathrm{Tl}$, we can see that the refined initial conditions with the shell model (dotted lines) and the
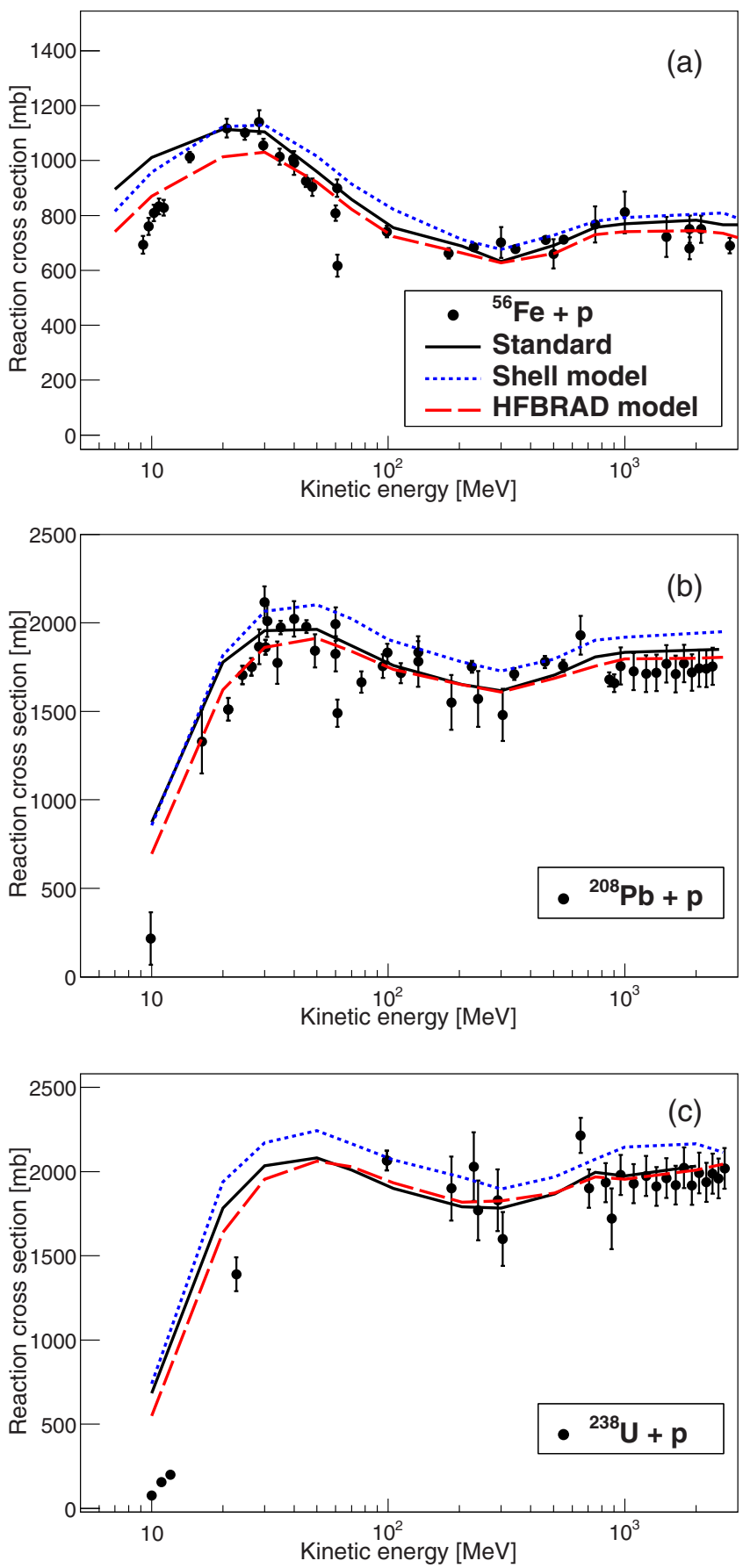

FIG. 12. Total reaction cross sections for proton-induced reactions as a function of the kinetic energy for different nuclei such as ${ }^{56} \mathrm{Fe}(\mathrm{a}),{ }^{208} \mathrm{~Pb}(\mathrm{~b})$, and ${ }^{238} \mathrm{U}$ (c). The solid line corresponds to standard INCL calculations while the dashed line represents calculations considering the best-fit parameters of the HFBRAD density profile. Experimental data taken from Refs. [55,77,91].

HFBRAD model (dashed lines) give similar results. Apart from the improvement of the cross sections for one-proton $\left({ }^{207} \mathrm{Tl}\right)$ and one-neutron removal $\left({ }^{207} \mathrm{~Pb}\right)$, the refined initial conditions also ameliorate the cross sections of lead and bismuth isotopes, where the new refined initial conditions 


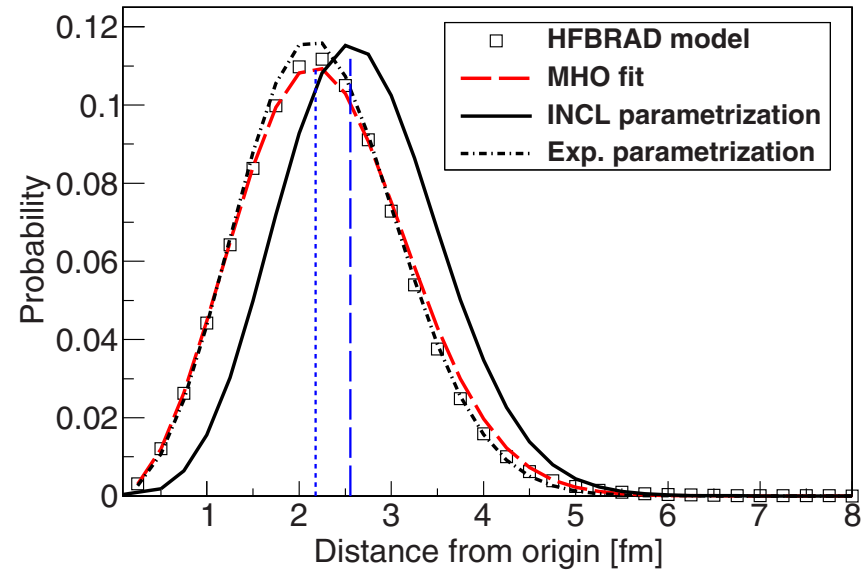

FIG. 13. Nuclear density probability for ${ }^{12} \mathrm{C}$. The open squares show the density calculated with the HFBRAD model. The dashed and solid lines correspond to the best-fit with the modified-harmonicoscilator (MHO) approximation [see Eq. (1)] and the results from the parametrization implemented in the standard INCL, respectively. The dot-dashed line is the MHO approximation with the experimental parameters taken from Ref. [45]. The vertical dashed and dotted lines are to indicate the average value of the standard INCL distribution and the other ones, respectively.

according to the HFBRAD model (dashed lines) provide cross sections closer to the experimental ones.

In Fig. 11, we also compare our new calculations for the heaviest residues produced in the reaction $p+{ }^{136} \mathrm{Xe}$ at $1 \mathrm{GeV}$ [81], where similar results are observed. We can see that the refined treatment according to the HFBRAD calculations (dashed lines) provides a better description of the cross sections for one-proton $\left({ }^{135} \mathrm{I}\right)$ and one-neutron removal $\left({ }^{135} \mathrm{Xe}\right)$ and also improves the production cross sections of cesium isotopes. In general, the refined treatment of the surface considerably improves the predictions for these cross sections.

\section{Total reaction cross sections}

This observable is determined by the intranuclear cascade stage and even by the first collision. From the Glauber-model picture, total reaction cross sections depend on the $N N$ total cross sections and on the target and projectile nuclear densities. Different benchmarks were performed with INCL for protonand neutron-induced reactions on various targets from ${ }^{9} \mathrm{Be}$ to ${ }^{238} \mathrm{U}$ [42], resulting in good agreement with experimental data for a large range of energies. However, in the present work, the parameters for the nuclear density distributions have been modified according to the HFBRAD model calculations and thus we have to benchmark the new approach. Moreover, INCL was not still validated completely for light-ion-induced reactions.

In Fig. 12, we overlap our new approaches and the standard one with experimental total reaction cross sections for protoninduced reactions on ${ }^{56} \mathrm{Fe}$ [Fig. 12(a)], ${ }^{208} \mathrm{~Pb}$ [Fig. 12(b)], and ${ }^{238} \mathrm{U}$ [Fig. 12(c)]. We can see that the calculations based on the phenomenological shell model used in our previous work [26] mostly overestimate the reaction cross sections for
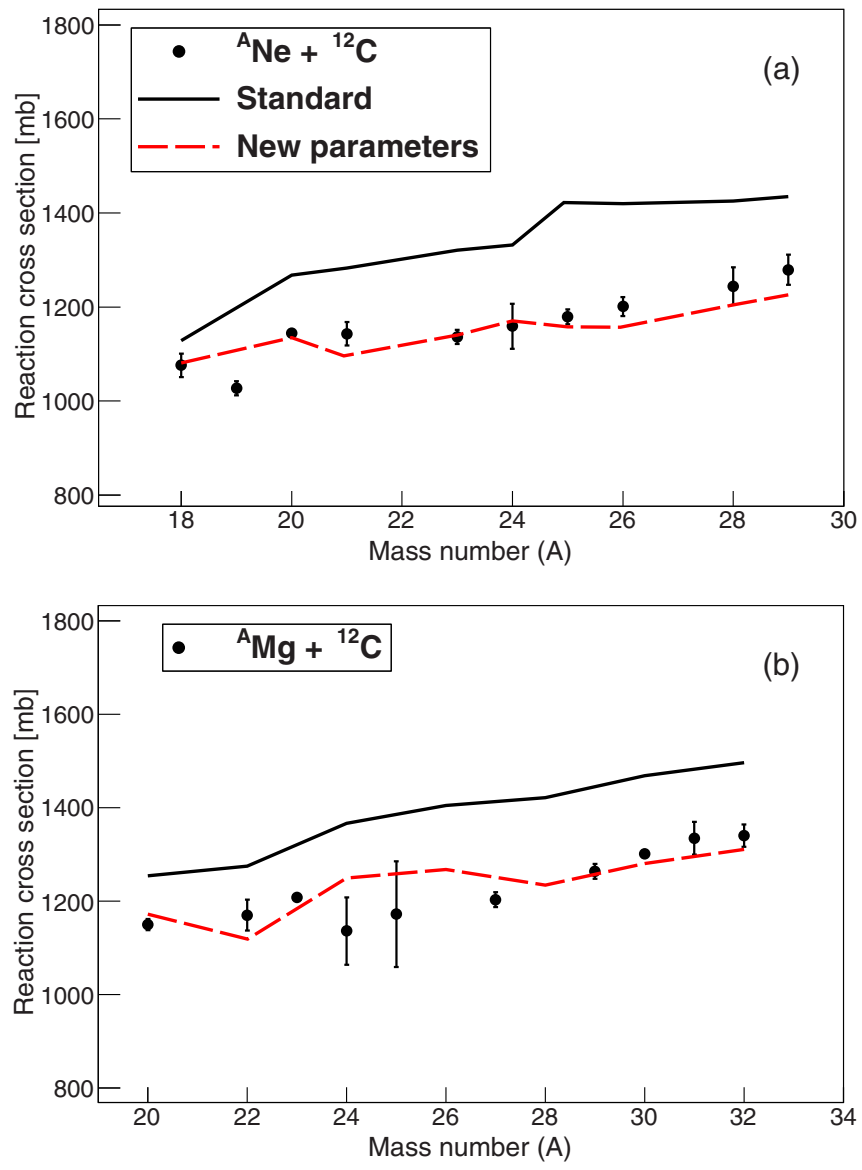

FIG. 14. Total reaction cross sections for different neon (a) and magnesium (b) isotopes impinging on a carbon target at $950 \mathrm{~A} \mathrm{MeV}$. Experimental data taken from Ref. [92]. The solid line corresponds to the standard preparation of light-ion projectiles in INCL while the dashed line represents calculations considering the best-fit parameters of the HFBRAD density profile with the MHO approximation.

energies above $50 \mathrm{MeV}$, whereas standard INCL and HFBRAD calculations give similar results for projectile kinetic energies above $50 \mathrm{MeV}$. Below this value, the new approach provides lower reaction cross sections. This difference appears because in the new approach the nucleons at the surface of the nucleus could be found deeper in the nuclear potential well [26] and this basic fact decreases the probability of removing nucleons and, consequently, it also reduces the total reaction cross sections. Note that the calculations performed with the new approach are a bit closer to the experimental data for kinetic energies below $20 \mathrm{MeV}$, improving the INCL predictions.

The good description of total reaction cross sections for nucleon-induced reactions is a clear proof about the correct implementation of $N N$ total cross sections and of target densities in INCL. Now, we will check the projectile preparation, whose details can be found in Ref. [41].

A simple way to test the preparation of light-ion projectiles is to draw the nuclear density probability as a function of the radius or the distance from origin, as shown in Fig. 13. The squares correspond to HFBRAD calculations for the nuclear 

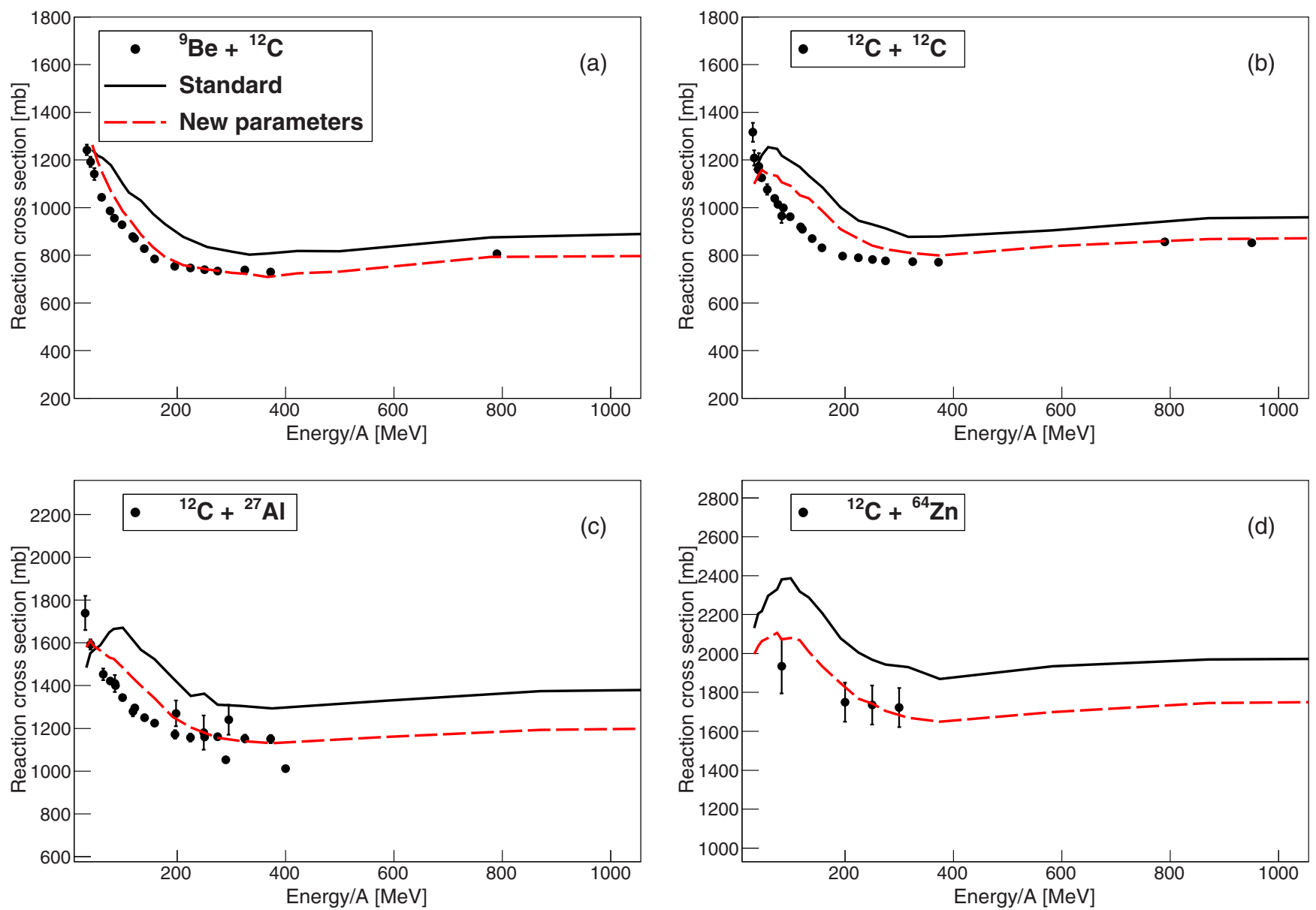

FIG. 15. Total reaction cross sections for the reactions ${ }^{9} \mathrm{Be}+{ }^{12} \mathrm{C}(\mathrm{a}),{ }^{12} \mathrm{C}+{ }^{12} \mathrm{C}(\mathrm{b}),{ }^{12} \mathrm{C}+{ }^{27} \mathrm{Al}$ (c), and ${ }^{12} \mathrm{C}+{ }^{64} \mathrm{Zn}(\mathrm{d})$ as a function of the projectile kinetic energy per nucleon. Experimental data taken from Refs. [92,93]. The solid line corresponds to the standard preparation of light-ion projectiles in INCL while the dashed line represents calculations considering the best-fit parameters of the HFBRAD density profile with the MHO approximation.

density probability of ${ }^{12} \mathrm{C}$ and the dashed line represents its best fit with the MHO approximation, $a=1.72 \mathrm{fm}$ and $\alpha=0.84$ [see Eq. (1)]. The solid and dot-dashed lines correspond to the results of the parametrization used in INCL and to the experimental parametrization of Ref. [45], respectively. One can note that the HFBRAD density probabilities are in good agreement with the experimental parametrization. Moreover, looking at the average values of the distributions (vertical dotted lines), we can also see that the standard INCL provides a density profile with a larger radius. This fact is observed in general for all light-ion projectiles (not shown). This overestimation of the projectile radius should increase artificially the total reaction cross sections for lightion-induced reactions.

The effect of these parametrizations on the total reaction cross sections is illustrated in Figs. 14 and 15. Reaction cross sections induced in light and medium nuclei by carbon or beryllium ions are displayed as a function of the projectile mass number in Figs. 14(a) and 14(b), and also as a function of the projectile kinetic energy in Figs. 15(a)-15(d) (solid circles). We can see that the INCL parametrization (solid lines) provides higher reaction cross sections due to the overestimation of the projectile radius. Note that this overestimation is systematic for all the reactions and there is no dependence on target mass number. Fixing the MHO density distribution improves the total reaction cross sections for light nuclei; however, we know that we should overestimate the light-ion-induced reaction cross section because we are neglecting Pauli blocking in the projectile. It is hard to quantify the effect without actually defining an implementation of Pauli blocking in the projectile, but hand-waving arguments suggest that it should be especially conspicuous in reactions between two light nuclei and at low energy. Inspection of Fig. 15 seems to indicate that this is indeed the case. Further investigations could be performed in this sense to improve the INCL model at low energies.

\section{SUMMARY AND CONCLUSIONS}

In our previous work, the initial conditions of the Liège intranuclear-cascade model (INCL) were improved using a refined description of the matter and energy densities at the nuclear surface on the basis of the shell model. This approach was applied to two nuclei, ${ }^{40} \mathrm{Ca}$ and ${ }^{208} \mathrm{~Pb}$, demonstrating that the new refined conditions ameliorate the description of 
one-nucleon-removal cross sections. Following these ideas, in the present work the initial conditions of INCL have been improved within Hartree-Fock-Bogoliubov calculations based on the HFBRAD model instead of the shell model. The initial refined conditions were introduced for all existing nuclei. The proton and neutron density profiles have been calculated with the HFBRAD model for all nuclei between the proton and neutron drip lines. We have also utilized these calculations to constrain the energy fluctuations at the nuclear surface. The new refined initial conditions lead to an increase of excitation energy for peripheral reactions.

We have seen that the one-nucleon-removal cross sections and the production cross sections of the heaviest residues can be reproduced rather well for proton-induced reactions on light, medium, and heavy targets by taking into account the presence of the neutron skin and the surface fuzziness. The use of Hartree-Fock-Bogoliubov calculations to shape the surface of the nucleus together with the capacity of INCL model to describe nucleon-nucleon collisions allow us to reproduce the mass and projectile energy dependencies of the one-nucleon-removal cross sections very well, especially for the one-proton-removal channel. Unfortunately, these benchmarks are performed with experimental data for stable nuclei only because there are no available data for nuclei far from stability.

Our calculations were also benchmarked for the first time with quadruple-differential cross sections for the reactions ${ }^{40} \mathrm{Ca}\left(p, p^{\prime} p^{\prime \prime}\right) \mathrm{X}$ and ${ }^{197} \mathrm{Au}\left(p, p^{\prime} p^{\prime \prime}\right) \mathrm{X}$ at 392 and $200 \mathrm{MeV}$, respectively, since the new refined conditions could affect the kinematics of the outgoing particles. However, we have seen that this observable is reproduced rather well by the standard version of INCL and that the new approaches do not introduce sizable differences. Therefore, we conclude that this experimental observable is mostly governed by the nucleonnucleon kinematics and by the rescattering of the particles involved in the collision. On the other hand, triple-differential cross sections for the reaction ${ }^{197} \mathrm{Au}(p, 2 p){ }^{196} \mathrm{Pt}$ at $200 \mathrm{MeV}$ are more sensitive to the new ingredients included in INCL and allow us to demonstrate that HFBRAD calculations describe the nuclear surface better than the shell model.
Total reaction cross sections induced by protons were also checked for reference nuclei, such as ${ }^{56} \mathrm{Fe},{ }^{208} \mathrm{~Pb}$, and ${ }^{238} \mathrm{U}$, because the new improvements could change the nuclear radius. In this case, we have seen that the phenomenological shell model used in our previous work [26] overestimates this observable systematically. Whereas calculations based on the HFBRAD model provide results that are in a better agreement with the experimental data, in particular, it seems to improve the description of the experimental data at energies below $20 \mathrm{MeV}$.

Finally, the preparation of light-ion projectiles in nucleusnucleus collisions has been also checked. We have found a small deviation in the standard INCL parametrizations with respect to Hartree-Fock-Bogoliubov calculations and experimental findings. The new parameters allow us to get a good agreement with the available experimental data of total reaction cross sections for nucleus-nucleus collisions at energies above $200 \mathrm{MeV}$. Below this energy, we also ameliorate the description of the experimental data with respect to standard INCL calculations, but more studies are required to get full agreement.

These improvements of INCL were done in the framework of the European nuclear science and application research program (ENSAR2), whose aim is to benchmark, validate, and provide model calculations with a high predictive power of experimental observables. The results presented in this work, together with the possibility of using this model in GEANT4 simulations [94], will facilitate the design of new experiments in different facilities, such as GSI/FAIR [59,95,96] and RIKEN [97,98], where kinematically complete measurements of quasifree knockout reactions of a broad range of nuclei impinging on hydrogen targets are proposed to investigate nuclear structure. Future benchmarks of INCL could be performed using experimental data from those experiments in order to provide more knowledge on this reaction.

\section{ACKNOWLEDGMENT}

This work has been partially supported by the EU ENSAR2 FP7 project (Grant Agreement No. 654002).
[1] R. Serber, Phys. Rev. 72, 1114 (1947).

[2] A. Boudard, J. Cugnon, S. Leray, and C. Volant, Phys. Rev. C 66, 044615 (2002).

[3] D. Mancusi, R. J. Charity, and J. Cugnon, Phys. Rev. C 82, 044610 (2010).

[4] E. A. Remler and A. P. Sathe, Ann. Phys. 91, 295 (1975).

[5] O. Buss, T. Gaitanos, K. Gallmeister, H. van Hees, M. Kaskulov, O. Lalakulich, A. B. Larionov, T. Leitner, J. Weil, and U. Mosel, Phys. Rep. 512, 1 (2012).

[6] Q. Li, J. Q. Wu, and C. M. Ko, Phys. Rev. C 39, 849 (1989).

[7] B. Blaettel, V. Koch, and U. Mosel, Rep. Prog. Phys. 56, 1 (1993).

[8] H. Sorge et al., Ann. Phys. 192, 266 (1989).

[9] J. Aichelin, Phys. Rep. 202, 233 (1991).

[10] K. Niita, S. Chiba, T. Maruyama, T. Maruyama, H. Takada, T. Fukahori, Y. Nakahara, and A. Iwamoto, Phys. Rev. C 52, 2620 (1995).
[11] M. Gonçalves, S. de Pina, D. A. Lima, W. Milomen, E. L. Medeiros, and S. B. Duarte, Phys. Lett. B 406, 1 (1997).

[12] G. Peilert, J. Konopka, H. Stöcker, W. Greiner, M. Blann, and M. G. Mustafa, Phys. Rev. C 46, 1457 (1992).

[13] V. E. Bunakov and G. V. Matvejev, Z. Phys. A 322, 511 (1985).

[14] D. F. Jackson, Nuclear Reactions (Methuen, London, 1970).

[15] P. Fröbrich, R. Lipperheide, and P. E. Hodgson, Theory of Nuclear Reactions, Oxford Studies in Nuclear Physics (Oxford University Press, Oxford, UK, 1996).

[16] N. S. Chant and P. G. Roos, Phys. Rev. C 15, 57 (1977).

[17] A. A. Cowley, G. J. Arendse, R. F. Visser, G. F. Steyn, S. V. Förtsch, J. J. Lawrie, J. V. Pilcher, T. Noro, T. Baba, K. Hatanaka et al., Phys. Rev. C 57, 3185 (1998).

[18] D. Filges and F. Goldenbaum, Handbook of Spallation Research: Theory, Experiments, and Applications (Wiley-VCH, Berlin, 2009). 
[19] J. Cugnon, C. Volant, and S. Vuillier, Nucl. Phys. A 620, 475 (1997).

[20] D. Mancusi, A. Boudard, J. Cugnon, J.-C. David, T. Gorbinet, and S. Leray, Phys. Rev. C 84, 064615 (2011).

[21] S. Leray, J.-C. David, M. Khandaker, G. Mank, A. Mengoni, N. Otsuka, D. Filges, F. Gallmeier, A. Konobeyev, and R. Michel, J. Korean Phys. Soc. 59, 791 (2011).

[22] J.-C. David, Eur. Phys. J. A 51, 68 (2015).

[23] B. Braunn, A. Boudard, J.-C. David, A. J. Koning, A. Leprince, S. Leray, and D. Mancusi, Eur. Phys. J. Plus 130, 153 (2015).

[24] C. Louchart, A. Obertelli, A. Boudard, and F. Flavigny, Phys. Rev. C 83, 011601(R) (2011).

[25] Y. L. Sun, J. Lee, Y. L. Ye, A. Obertelli, Z. H. Li, N. Aoi, H. J. Ong, Y. Ayyad, C. A. Bertulani, J. Chen et al., Phys. Rev. C 93, 044607 (2016).

[26] D. Mancusi, A. Boudard, J. Carbonell, J. Cugnon, J.-C. David, and S. Leray, Phys. Rev. C 91, 034602 (2015).

[27] L. Audirac, A. Obertelli, P. Doornenbal, D. Mancusi, S. Takeuchi, N. Aoi, H. Baba, S. Boissinot, A. Boudard, A. Corsi et al., Phys. Rev. C 88, 041602 (2013).

[28] T. E. Rodrigues, J. D. T. Arruda-Neto, A. Deppman, V. P. Likhachev, J. Mesa, C. Garcia, K. Shtejer, G. Silva, S. B. Duarte, and O. A. P. Tavares, Phys. Rev. C 69, 064611 (2004).

[29] T. E. Rodrigues, M. N. Martins, C. Garcia, J. D. T. Arruda-Neto, J. Mesa, K. Shtejer, and F. Garcia, Phys. Rev. C 75, 014605 (2007).

[30] T. E. Rodrigues, J. D. T. Arruda-Neto, J. Mesa, C. Garcia, K. Shtejer, D. Dale, I. Nakagawa, and P. L. Cole, Phys. Rev. Lett. 101, 012301 (2008).

[31] K. Bennaceur and J. Dobaczewski, Comput. Phys. Commun. 168, 96 (2005).

[32] N. Metropolis, R. Bivins, M. Storm, A. Turkevich, J. M. Miller, and G. Friedlander, Phys. Rev. 110, 185 (1958); N. Metropolis, R. Bivins, M. Storm, Anthony Turkevich, J. M. Miller, and G. Friedlander, ibid. 110, 204 (1958).

[33] H. W. Bertini, Phys. Rev. 131, 1801 (1963); 188, 1711 (1969).

[34] K. Chen, Z. Fraenkel, G. Friedlander, J. R. Grover, J. M. Miller, and Y. Shimamoto, Phys. Rev. 166, 949 (1968).

[35] Y. Yariv and Z. Fraenkel, Phys. Rev. C 20, 2227 (1979).

[36] V. S. Barashenkov, B. F. Kostenko, and A. M. Zadorogny, Nucl. Phys. A 338, 413 (1980).

[37] J. Cugnon, Phys. Rev. C 23, 2094 (1981).

[38] T. Kodama, S. B. Duarte, K. C. Chung, and R. A. M. S. Nazareth, Phys. Rev. Lett. 49, 536 (1982).

[39] K. K. Gudima, S. G. Mashnik, and V. D. Toneev, Nucl. Phys. A 401, 329 (1983).

[40] H. Duarte, Phys. Rev. C 75, 024611 (2007).

[41] D. Mancusi, A. Boudard, J. Cugnon, J.-C. David, P. Kaitaniemi, and S. Leray, Phys. Rev. C 90, 054602 (2014).

[42] A. Boudard, J. Cugnon, J.-C. David, S. Leray, and D. Mancusi, Phys. Rev. C 87, 014606 (2013).

[43] J. Cugnon and P. Henrotte, Eur. Phys. J. A 16, 393 (2003).

[44] T. Aoust and J. Cugnon, Phys. Rev. C 74, 064607 (2006).

[45] H. D. Vries, C. W. D. Jager, and C. D. Vries, At. Data Nucl. Data Tables 36, 495 (1987).

[46] J. Cugnon, D. L'Hôte, and J. Vandermeulen, Nucl. Instrum. Methods Phys. Res., Sect. B 111, 215 (1996).

[47] A. Kelić, M. V. Ricciardi, and K.-H. Schmidt, in Proceedings of Joint ICTP-IAEA Advanced Workshop on Model Codes for Spallation Reactions, ICTP Trieste, Italy, 4-8 February 2008, edited by D. Filges, S. Leray, Y. Yariv, A. Men- goni, A. Stanculescu, and G. Mank (IAEA, Vienna, 2008), pp. 181-221.

[48] V. F. Weisskopf and D. H. Ewing, Phys. Rev. 57, 472 (1940).

[49] A. H. Wapstra, G. Audi, and C. Thibault, Nucl. Phys. A 729, 129 (2003).

[50] R. Bass, Proceedings of the Symposium on Deep-Inelastic and Fusion Reactions with Heavy Ions (Springer Verlag, Berlin, 1980).

[51] A. V. Ignatyuk, Proceedings of the Conference Bologna 2000: Structure of the Nucleus at the Dawn of the Century, Bologna, Italy, 29 May - 3 June 2000, edited by G. C. Bonsignori, M. Bruno, A. Ventura, and D. Vretenar (World Scientific, Singapore, 2001), pp. 287-292.

[52] B. Jurado, K.-H. Schmidt, and J. Benlliure, Phys. Lett. B 553, 186 (2003).

[53] B. Jurado, C. Schmitt, K.-H. Schmidt, J. Benlliure, and A. R. Junghans, Nucl. Phys. A 747, 14 (2005).

[54] M. V. Ricciardi, P. Armbruster, J. Benlliure, M. Bernas, A. Boudard, S. Czajkowski, T. Enqvist, A. Kelić, S. Leray, R. Legrain et al., Phys. Rev. C 73, 014607 (2006).

[55] C. Villagrasa-Canton, A. Boudard, J.-E. Ducret, B. Fernandez, S. Leray, C. Volant, P. Armbruster, T. Enqvist, F. Hammache, K. Helariutta et al., Phys. Rev. C 75, 044603 (2007).

[56] J. Alcántara-Núñez, J. Benlliure, C. Paradela, C. Pérez-Loureiro, J. L. Rodríguez-Sánchez, L. Audouin, A. Boudard, E. Casarejos, T. Enqvist, F. Farget et al., Phys. Rev. C 92, 024607 (2015).

[57] Y. Ayyad, J. Benlliure, J. L. Rodríguez-Sánchez, A. Bacquias, A. Boudard, E. Casarejos, T. Enqvist, M. Fernandez, V. Henzl, V. Henzlova et al., Phys. Rev. C 91, 034601 (2015).

[58] J. L. Rodríguez-Sánchez et al. (SOFIA Collaboration), Phys. Rev. C 91, 064616 (2015); 92, 044612 (2015); 94, 061601(R) (2016)

[59] J. Benlliure and J. L. Rodríguez-Sánchez, Eur. Phys. J. Plus 132, 120 (2017).

[60] M. Fidelus, D. Filges, F. Goldenbaum, H. Hodde, A. Jany, L. Jarczyk, B. Kamys, M. Kistryn, S. Kistryn, S. Kliczewski et al., Phys. Rev. C 89, 054617 (2014).

[61] Y. Ayyad, J. Benlliure, E. Casarejos, H. Alvarez-Pol, A. Bacquias, A. Boudard, M. Caamaño, T. Enqvist, V. Föhr, A. Kelić-Heil et al., Phys. Rev. C 89, 054610 (2014).

[62] J. L. Rodríguez-Sánchez et al. (SOFIA Collaboration), Phys. Rev. C 90, 064606 (2014).

[63] S. Lo Meo, D. Mancusi, C. Massimi, G. Vannini, and A. Ventura, Nucl. Phys. A 933, 43 (2015).

[64] S. Pedoux, J. Cugnon, A. Boudard, J.-C. David, and S. Leray, Adv. Space Res. 44, 926 (2009).

[65] D. Mancusi, S. Lo Meo, N. Colonna, A. Boudard, M. A. CortésGiraldo, J. Cugnon, J.-C. David, S. Leray, J. Lerendegui-Marco, C. Massimi, and V. Vlachoudis, Eur. Phys. J. A 53, 80 (2017).

[66] E. Chabanat, P. Bonche, P. Haensel, J. Meyer, and R. Schaeffer, Nucl. Phys. A 635, 231 (1998).

[67] S. Terashima, H. Sakaguchi, H. Takeda, T. Ishikawa, M. Itoh, T. Kawabata, T. Murakami, M. Uchida, Y. Yasuda, M. Yosoi et al., Phys. Rev. C 77, 024317 (2008).

[68] V. Lapoux and N. Alamanos, Eur. Phys. J. A 51, 91 (2015).

[69] D. N. Basu, Int. J. Mod. Phys. E 13, 747 (2004).

[70] K.-H. Schmidt et al. (FRS Collaboration), Nucl. Phys. A 542, 699 (1992).

[71] D. Perez-Loureiro, J. Benlliure, H. Alvarez-Pol, B. Blank, E. Casarejos, D. Dragosavac, V. Föhr, M. Gascón, W. Gawlikowicz, A. Heinz et al., Phys. Lett. B 703, 552 (2011). 
[72] T. Kurtukian-Nieto, J. Benlliure, K.-H. Schmidt, L. Audouin, F. Becker, B. Blank, E. Casarejos, F. Farget, M. FernándezOrdóñez, J. Giovinazzo et al., Phys. Rev. C 89, 024616 (2014).

[73] K. Amos, S. Karataglidis, and J. Dobaczewski, Phys. Rev. C 70, 024607 (2004).

[74] C.-X. Chen, S. Albergo, Z. Caccia, S. Costa, H. J. Crawford, M. Cronqvist, J. Engelage, L. Greiner, T. G. Guzik, A. Insolia et al., Phys. Rev. C 56, 1536 (1997).

[75] W. R. Webber, J. C. Kish, and D. A. Schrier, Phys. Rev. C 41, 547 (1990).

[76] L. Audouin et al. (CHARMS Collaboration), Nucl. Phys. A 768, 1 (2006).

[77] T. Enqvist et al. (CHARMS Collaboration), Nucl. Phys. A 686, 481 (2001).

[78] A. S. Iljinov, V. G. Semenov, M. P. Semenova, N. M. Sobolevsky, and L. V. Udovenko, Production of Radionuclides at Intermediate Energies, Landolt-Bornstein New Series Vol. I/13 (Springer-Verlag, Berlin, 1991).

[79] D. L. Olson, B. L. Berman, D. E. Greiner, H. H. Heckman, P. J. Lindstrom, and H. J. Crawford, Phys. Rev. C 28, 1602 (1983).

[80] W. R. Webber, J. C. Kish, J. M. Rockstroh, Y. Cassagnou, R. Legrain, A. Soutoul, O. Testard, and C. Tull, Astrophys. J. 508, 940 (1998).

[81] P. Napolitani, K.-H. Schmidt, L. Tassan-Got, P. Armbruster, T. Enqvist, A. Heinz, V. Henzl, D. Henzlova, A. Kelić, R. Pleskac et al., Phys. Rev. C 76, 064609 (2007).

[82] L. Giot et al. (CHARMS Collaboration), Nucl. Phys. A 899, 116 (2013).

[83] C. Paradela, L. Tassan-Got, J. Benlliure, J. L. RodríguezSánchez, L. Audouin, A. Boudard, E. Casarejos, T. Enqvist, J. E. Ducret, F. Farget et al., Phys. Rev. C 95, 044606 (2017).
[84] N. P. Jacob and S. S. Markowitz, Phys. Rev. C 11, 541 (1975).

[85] F. Rejmund et al. (CHARMS Collaboration), Nucl. Phys. A 683, 540 (2001).

[86] J. Taïeb et al. (CHARMS Collaboration), Nucl. Phys. A 724, 413 (2003).

[87] S. Meloni and J. B. Cumming, Phys. Rev. 136, B1359 (1964).

[88] S. V. Förtsch, A. A. Cowley, J. J. Lawrie, J. V. Pilcher, F. D. Smit, and D. M. Whittal, Phys. Rev. C 48, 743 (1993).

[89] A. A. Cowley, G. F. Steyn, Y. Watanabe, T. Noro, K. Tamura, M. Kawabata, K. Hatanaka, H. Sakaguchi, H. Takeda, and M. Itoh, Phys. Rev. C 62, 064604 (2000).

[90] A. Kelić, K.-H. Schmidt, T. Enqvist, A. Boudard, P. Armbruster, J. Benlliure, M. Bernas, S. Czajkowski, R. Legrain, S. Leray et al., Phys. Rev. C 70, 064608 (2004).

[91] R. F. Carlson, At. Data Nucl. Data Tables 63, 93 (1996).

[92] A. Ozawa, T. Suzuki, and I. Tanihata, Nucl. Phys. A 693, 32 (2001).

[93] M. Takechi, M. Fukuda, M. Mihara, K. Tanaka, T. Chinda, T. Matsumasa, M. Nishimoto, R. Matsumiya, Y. Nakashima, H. Matsubara et al., Phys. Rev. C 79, 061601(R) (2009).

[94] http://geant4.cern.ch/

[95] T. Aumann, C. A. Bertulani, and J. Ryckebusch, Phys. Rev. C 88, 064610 (2013).

[96] J. L. Rodríguez-Sánchez, J. Benlliure, C. A. Bertulani, J. Vargas, Y. Ayyad, H. Alvarez-Pol, J. Atkinson, T. Aumann, S. BeceiroNovo, K. Boretzky et al., Phys. Rev. C 96, 034303 (2017).

[97] A. Obertelli, A. Delbart, S. Anvar, L. Audirac, G. Authelet, H. Baba, B. Bruyneel, D. Calvet, F. Château, A. Corsi et al., Eur. Phys. J. A 50, 8 (2014).

[98] A. Obertelli, Eur. Phys. J. Plus 131, 319 (2016). 